\title{
Les enclos du haut Moyen Âge de Vauvert à Château-Gontier (Mayenne)
}

The Vauvert Early Mediaeval Enclosures at Château-Gontier (Mayenne)

Alain Valais, Rémy Arthuis, Jean-François Nauleau et Isabelle MoréraVinçotte

\section{Q OpenEdition}

\section{Journals}

Édition électronique

URL : https://journals.openedition.org/rao/898

DOI : $10.4000 /$ rao.898

ISBN : 978-2-7535-1609-0

ISSN : 1775-3732

Éditeur

Presses universitaires de Rennes

Édition imprimée

Date de publication : 31 décembre 2009

Pagination : 205-227

ISBN : 978-2-7535-1086-9

ISSN : 0767-709X

\section{Référence électronique}

Alain Valais, Rémy Arthuis, Jean-François Nauleau et Isabelle Moréra-Vinçotte, "Les enclos du haut Moyen Âge de Vauvert à Château-Gontier (Mayenne) », Revue archéologique de l'Ouest [En ligne], 26 | 2009, mis en ligne le 31 décembre 2011, consulté le 22 août 2022. URL : http:// journals.openedition.org/rao/898; DOl : https://doi.org/10.4000/rao.898 


\title{
Les enclos du haut Moyen Âge de Vauvert à Château-Gontier (Mayenne)
}

\author{
The Vauvert Early Mediaeval Enclosures at Château-Gontier (Mayenne)
}

\author{
Alain VALAIS * \\ avec la collaboration de Rémy Arthuis ${ }^{* *}$, Jean-François Nauleau ** \\ et Isabelle MorérA-VinçOTTE ***
}

Résumé : Le site de Vauvert, repéré d’avion par G. Leroux en 1992, se compose de fossés pour la plupart profondément creusés dans les schistes précambriens. Ils limitent plusieurs parcelles carrées ou rectangulaires. La plus ancienne, établie à la fin du VI ${ }^{\mathrm{e}}$ siècle ou au siècle suivant constitue sans doute le noyau initial du site. Plusieurs parcelles vont venir progressivement entourer cet enclos primitif dont nous n'avons pu fouiller qu'une petite partie, le reste ayant été détruit juste avant l'évaluation de J.-C. Meuret en 1999.

Les structures d'habitat découvertes sont rares et souvent mal conservées. Elles se résument à un four domestique, une fosse complexe, quelques trous de poteau, des fosses-tranchées énigmatiques et une sépulture établie dans le fond d'un fossé. La partie explorée de ce site, qui appartient à la périphérie d'un habitat beaucoup plus vaste, n’est plus occupée après la période carolingienne.

\begin{abstract}
The Vauvert site, identified from the air by G. Leroux in 1992, consists of ditches most deeply cut in precambrian schists, which delimit several square rectangular plots. The earliest, established by the end of Vth century AD or during the following one, is probably the initial nucleus of the site. Several others progressively surround this basic enclosure; we could only excavate a small part, the rest having been destroyed just before evaluation by J.-C. Meuret in 1999.

The domestic structures are few and often ill-preserved: a domestic hearth, a complex pit, some postholes, some engmatic ditches and a grave placed in the bottom of a ditch. The explored part of the site, which belongs to the periphery of a much larger settlement, is no longer occupied after the Carolingian period.
\end{abstract}

Mots clés : Haut Moyen Âge, enclos, trous de poteaux, four, fosse aménagée, sépulture, céramique

Key words: Early Middle Ages, enclosure, postholes, oven, equiped pit, grave, ceramics.

\section{LOCALISATION DES SITES ET TOPOGRAPHIE DU SECTEUR}

Les enclos de Vauvert se situent à la périphérie sud-ouest de Château-Gontier sur la rive droite de la Mayenne, rivière dont ils sont distants de $1100 \mathrm{~m}$ (fig. 1). Implantés sur une proéminence (altitude $51 \mathrm{~m}$ ) les vestiges dominent depuis le sud-ouest la vallée du Ponceau (fig. 2). Le gisement constitue dans le secteur, avec la colline de Miliana toute proche (altitude $84 \mathrm{~m}$ ) et le site d'implantation de la ville médiévale de Château-Gontier, l'un des derniers points hauts avant la vallée de la Mayenne.

* INRAP - 9 rue Volney, 53200 Château-Gontier.

** INRAP - 4 rue du Tertre, 44477 Carquefou.

*** 65 rue des Drouillard, 44620 La Montagne. 


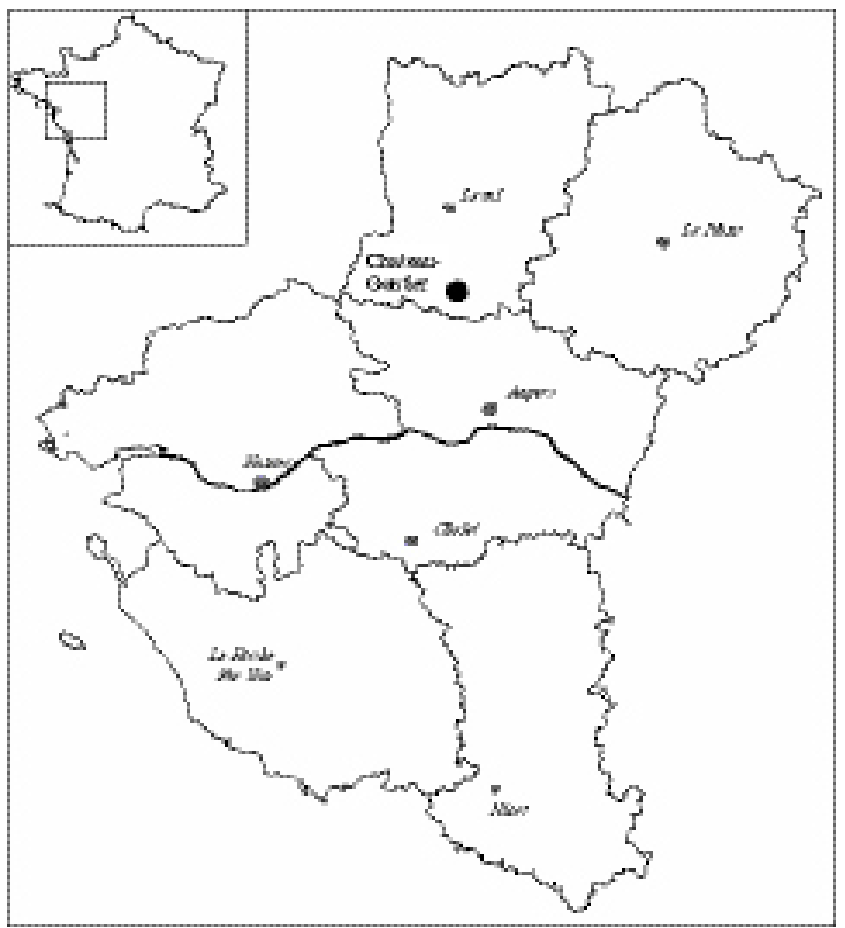

Figure 1 : Localisation générale et régionale.

Figure 1: General and regional location maps.

\section{Contexte géologique}

Le substrat se compose de schistes précambriens qui apparaissent sur la totalité de l'emprise fouillée à moins de $0,50 \mathrm{~m}$ sous la surface. Sur les points les plus hauts du site, la roche est seulement recouverte par 0,30 m d'humus mais, dès que l'on s'écarte du sommet, le substrat disparaît sous une couche de limons d'altération qui devient de plus en plus épaisse. La foliation du schiste est sub-verticale. Il est moyennement altéré et se délite en plaquettes de un à plusieurs centimètres. Ce schiste est décompressé, ce qui rend possible la circulation de l'eau le long des plans de foliation. Ce constat est confirmé par l'observation des sols labourés et des sédiments de remplissage des structures : aucune trace d'hydromorphie n'y est visible; les sols sont donc bien drainés grâce à une bonne circulation de l'eau dans le sous-sol et à des pentes naturelle propices au ressuyage.

\section{Contexte archéologique}

\section{La Protohistoire et l'Antiquité}

Les sites des Fosses ( $\mathrm{n}^{\circ}$ ) et de La Réhaudière (en dehors de la carte), sur la commune de Saint-Fort, constituent sans doute les traces les plus orientales de l'exploitation aurifere des « Miaules », ligne dont on retrouve les excavations jusqu'à
La Selle-Guerchaise en Ille-et-Vilaine (Meuret, 1993, p. 23; Naveau, 1992, p. 86). Cette ressource pourrait expliquer la profusion de sites gaulois et gallo-romains que G. Leroux détecte en prospection aérienne tout au long de cet axe depuis le début des années 90. La région de Château-Gontier n'y fait pas exception, puisqu'elle montre également de nombreux enclos. En 1975, la triple enceinte de la Mazure ( $\left.{ }^{\circ} 9\right)$, qui remonte à La Tène finale, en fut le premier exemplaire fouillé (Lambert et Riouffreyt, 1976). Le mobilier découvert à cette occasion témoigne plutôt d'activités domestiques; le matériel antique également recueilli indique qu'un site gallo-romain existe sans doute à proximité.

Non loin de la Mazure, à $2000 \mathrm{~m}$ environ, un autre site enclos, le Clos-Henry ( $\left.{ }^{\circ} 10\right)$, a été repéré tout récemment à moins de $200 \mathrm{~m}$ de Vauvert (Valais et al., 2008). L'indice en a été découvert fortuitement en 2000 et les sondages d'évaluation réalisés en même temps que la présente opération ont montré qu'il s'agissait des vestiges d'une ferme indigène de La Tène finale. La découverte en 2001 de plusieurs indices de sites de l'âge du Fer sur le tracé de la rocade nord de Château-Gontier/Bazouges, au nord des fermes de la Morlière $\left(\mathrm{n}^{\circ} 11\right)$ et de la Petite Jariais $\left(\mathrm{n}^{\circ} 12\right)$, confirme encore l'importance de l'occupation de la fin de l'âge du Fer autour de Château-Gontier (Langlois, 2001).

\section{Le haut Moyen Âge}

Les périodes antérieures à la fin de la protohistoire sont peu illustrées dans la région de Château-Gontier (fig. 2). On y dénombre néanmoins quelques découvertes isolées pour la période néolithique, notamment une fosse VSG sur le site voisin du Clos-Henry ( $\left.{ }^{\circ} 10\right)$, à moins de $200 \mathrm{~m}$ au sud-ouest de Vauvert (Valais et al., 2008), et un dolmen à la Pêcherie $\left(\mathrm{n}^{\circ} 1\right)$, sur la commune de Loigné. Il faut attendre la fin de La Tène pour voir se multiplier les découvertes. Loppidum de la Cadurie ( $\mathrm{n}^{\circ} 3$ ), qui occupe une douzaine d'hectares, domine la confluence de la Mayenne et du Bouillon aux confins des communes de Château-Gontier/ Bazouges et de Loigné (Angot, 1910, tome III, p. 123b); des monnaies romaines indiqueraient que ce site fortifié de La Tène finale a été occupé également durant la période gallo-romaine.

Les données archéologiques remontant au haut Moyen Âge se limitent à des indices funéraires, qui supposent malgré tout de l'existence d'habitats. Les nécropoles les plus proches sont situées à Loigné-sur-Mayenne avec les découvertes au $\mathrm{XIX}^{\mathrm{e}}$ siècle de sarcophages en calcaire coquillier et de coffres en schiste, à la fois à la Françoisière $\left(n^{\circ} 13\right)$ et à l'Érable ( $\left.{ }^{\circ} 14\right)$. D'autres sarcophages dont on ignore le type ont également été repérés autour de l'église du village (Naveau, 1992, p. 175). Des mentions du même ordre existent au nord de la zone, sur la commune de Saint-Sulpice, à 


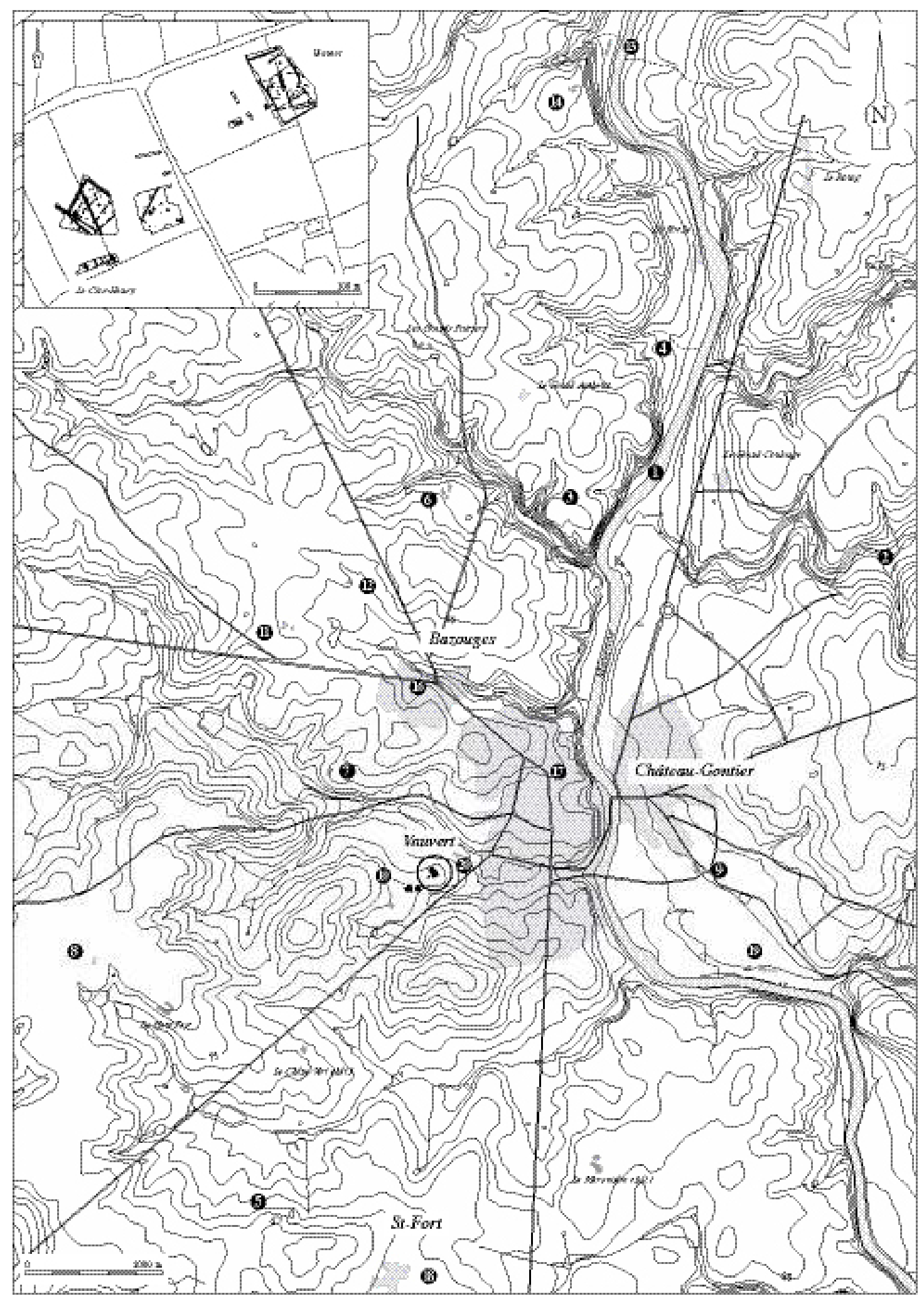

Figure 2 : Carte archéologique de la région de Château-Gontier et plan de détail montrant les sites de Vauvert et du Clos-Henry (les numéros renvoient au texte).

Figure 2: Archaeolgical map of Chatteau-Gontier surrounds and detail of the sites of Vauvert and Clos-Henry (numbers refer to the text). 
Neuville avec des cuves en calcaire coquillier et à la Querlière où elles sont associées à des coffres en schiste (Colleter, 2003, p. 186-187). Des inhumations des deux types ont également été mises en évidence au sud de Château-Gontier sous le village de Ménil (Bocquet et al., 2008).

Une inscription carolingienne a été découverte à Bazouges en 1835 ( $\left.\mathrm{n}^{\circ} 16\right)$; conservée dans l'église du village, c'est une plaque de schiste gravé, datée de 876 . Le texte indique "sous cette dalle, le corps de Giswahl, homme pieux et d'heureuse mémoire. Sa foi fut véritable et sa vie sainte. Il décéda ici, le 3 des calendes d'avril. "Toi qui viens ici et lis, qui que tu sois, dis je te prie: Dieu souverain, aie pitié de Giswhall, ton serviteur." L'année de l'incarnation du seigneur, 9ème indiction, sous la première année du règne de l'Empereur Charles" (Naveau, 1999, p. 118). Cet élément a été découvert juste à la sortie du bourg, lors de l'aménagement de la route de Laval. Sa présence indique au minimum qu'une nécropole carolingienne existait à peu de distance de l'église dédiée à Saint Martin, vocable ancien associé encore une fois à ce type de découverte. Des sondages archéologiques, réalisés autour de l'édifice de Bazouges, n’ont pourtant révélé aucune autre sépulture aussi ancienne (Mare, 1989).

\section{Les textes}

Les textes mentionnent également quelques implantations. Il existe un prieuré à Azé dès le $\mathrm{vI}^{\mathrm{e}}$ siècle (Legros, 2006, p. 35) et des villae à Laigné et à Ampoigné au Ix siècle (ibid., p. 37). La cour de Bazouges apparaît avant 1001, mais sans que l'on puisse exactement en évaluer l'importance (de Broussillon, 1903, acte $n^{\circ}$ 677). Toutefois, la cour d'Oudainville, contre laquelle elle est échangée, dispose de biens importants comme des terres, bois, prés, villulae, des moulins et deux églises (Legros, 2006, p. 39). Celle de Bazouges devait donc être d'importance comparable.

\section{Circonstance des découvertes et évaluation}

Le site de Vauvert a été repéré en prospection aérienne par G. Leroux en 1992 (fig. 3). Il s'agissait d'anomalies rectilignes indiquant l'existence d'un enclos sub-rectangulaire d'environ $3000 \mathrm{~m}^{2}$ à cloisonnements internes. Une interruption, du côté est, permettait de localiser une entrée. La partie nord du site montrait beaucoup plus d'imprécisions.

En 1998, la viabilisation du secteur en vue de la construction d'une zone pavillonnaire menace directement le site. Un zonage archéologique est alors défini en vue d'une opération préventive, réalisée en 1999 par J.-C. Meuret. Celle-ci avait pour but d'évaluer le site à l'aide de sondages (tranchées et petits décapages) mais également de procéder, en périphérie, à l'ouverture de tranchées de prospection (Meuret, 2003, p. 23-36). Malheureusement, un rond-point, une rue et des

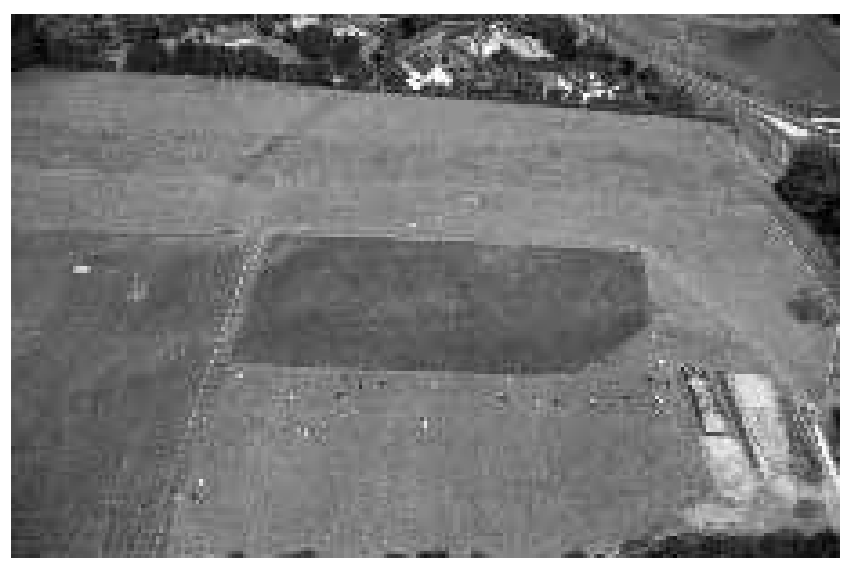

Figure 3 : Photographie aérienne du site en 1992 vue depuis l'ouest (cliché G. Leroux).

Figure 3: Air photograph taken from West in 1992.

réseaux (gaz et électricité) avaient déjà été établis quelques semaines plus tôt, à l'intérieur du périmètre archéologique (Meuret, 1999, p. 5). Ce point, nous allons le voir, aura de lourdes conséquences pour le site.

Lévaluation a permis de confirmer la présence d'un enclos dont deux côtés, au sud et à l'ouest, ont été retrouvés (fig. 4). Au nord, en revanche, les limites de son emprise n'ont pu être cernées en raison des travaux déjà réalisés. De manière assez inattendue, car on avait plutôt pronostiqué une occupation de La Tène, les sondages n'ont livré que du mobilier du haut Moyen Âge. Cette datation a été confirmée par une analyse radiocarbone réalisée sur des charbons de bois issus d'un des fossés; elle donne un âge calibré entre 655 et $799^{1}$. Devant l'intérêt de ces vestiges et de leur datation, la fouille du site a été programmée.

\section{LE SITE DU HaUt Moyen ÂGE}

\section{Des enclos multiples}

Le gisement présente une série d'enclos juxtaposés dont plusieurs sont incomplets (fig. 5). Pour plus de lisibilité dans l'eposé, les fossés ont été regroupés et feront l'objet d'une présentation par ensembles cohérents (long fossé rectiligne, ou fossés formant un enclos évident par exemple).

1. Code laboratoire LY 9603 1330+/-35BP, âge calibré de 655 à 769 apr. J.-C. Dates autour desquelles se situent le maximum de probabilités : 674 , 755, 705 après J.-C. (en probabilités décroissantes). 
Figure 4: Données de l'évaluation de J.-C. Meuret (1999) reportées sur les traces redressées de la photographie aérienne de G. Leroux.

Figure 4: Field evaluation data by J.-C. Meuret (1999) faced related with the adjusted air photograph.

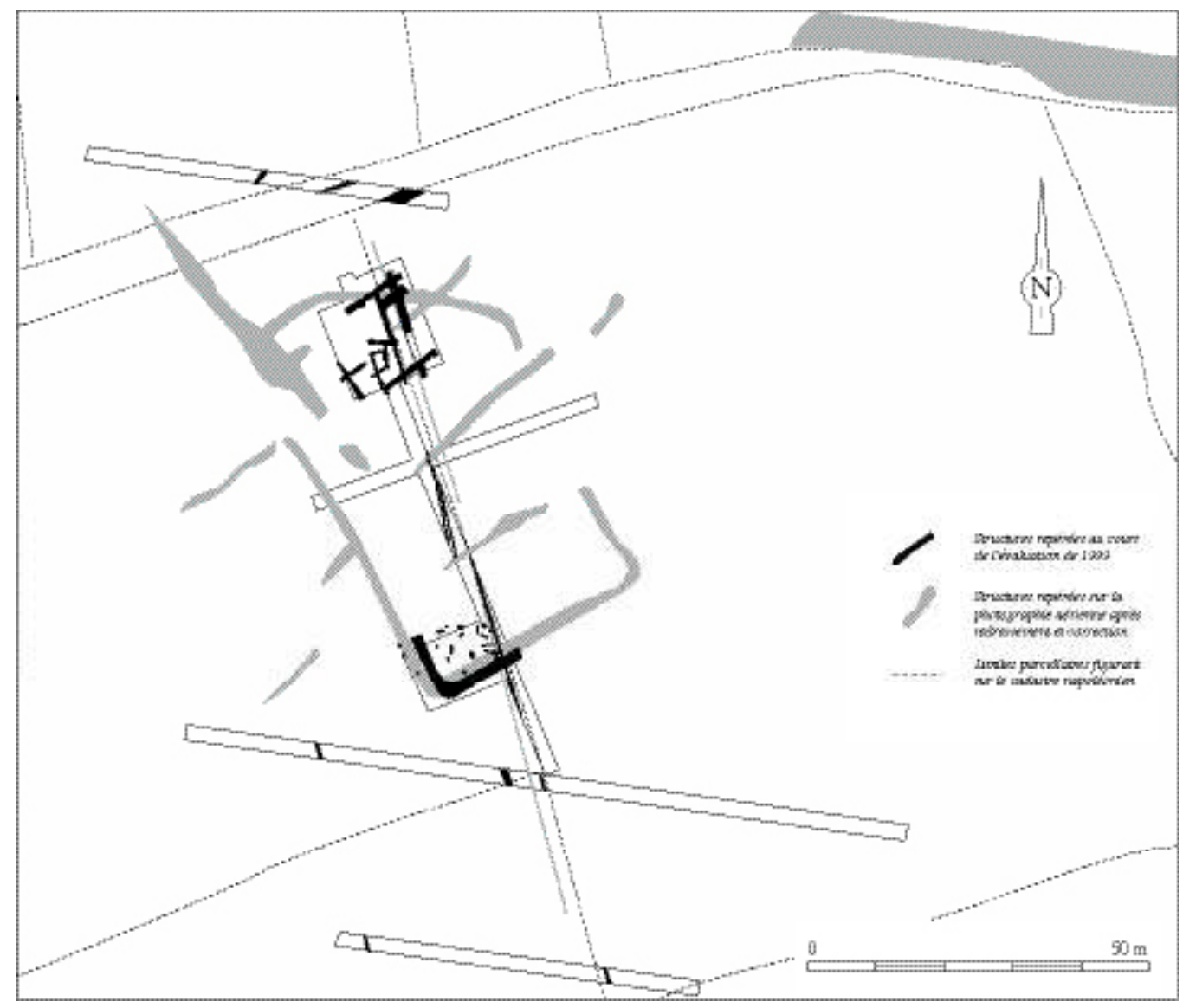

\section{Les ensembles du haut Moyen Âge ${ }^{2}$}

L'ensemble 1 appartient à la partie nord-ouest du décapage. Il est composé de deux fossés, F 112/101 et F 103 décrivant un angle droit. Son côté nord/sud a été testé à huit reprises sur les $27 \mathrm{~m}$ de son développement où il mesure le plus souvent $0,90 \mathrm{~m}$ de largeur pour $0,40 \mathrm{~m}$ de profondeur ${ }^{3}$. Son retour vers l'ouest suivi sur $6 \mathrm{~m}$, mesure jusqu'à $1,50 \mathrm{~m}$ de largeur et environ $0,50 \mathrm{~m}$ de profondeur. Notons que cette limite a été retrouvée beaucoup plus à l'ouest dans un sondage isolé.

L'ensemble 2 (F. 2) a été testé à quatre reprises lors de cette opération, et sur une douzaine de mètres lors de l'évaluation. Il est perpendiculaire à l'ensemble 1 (F 112) et mesure $27 \mathrm{~m}$ de longueur, entre 1,10 et $1,30 \mathrm{~m}$ de largeur pour $0,90 \mathrm{~m}$ de profondeur; son profil en $\mathrm{V}$ est remarquable.

L'ensemble 3, constitué par les fossés F 1 et F 116, a été suivi sur $45 \mathrm{~m}$. Avec un tracé rectiligne, il est d'abord parallèle au grand côté de l'ensemble 1 et s'infléchit ensuite légè-

2. Il s'agit ici des ensembles qui ont livré du mobilier significatif ou qui entrent a priori dans le système parcellaire du site.

3. Sauf précision contraire, toutes les profondeurs seront établies par rapport à la surface du décapage. rement vers l'ouest où il s'interrompt. Il devient de moins en moins important du sud au nord : à $1 \mathrm{~m}$ de largeur pour 0,50 à $0,65 \mathrm{~m}$ de profondeur durant les vingt premiers mètres, il ne fait plus ensuite que 0,60 à $0,70 \mathrm{~m}$ de largeur pour moins de $0,30 \mathrm{~m}$ de profondeur et, plus au nord, il ne mesure plus que $0,05 \mathrm{~m}$ de profondeur pour reprendre un peu d'importance avant de sortir de l'emprise étudiée. Il est probable qu'il y ait eu une interruption du fossé à cet endroit (en partie détruit par le passage d'une conduite de gaz). Au-delà, il dépasse à nouveau une trentaine de centimètres de profondeur pour $0,70 \mathrm{~m}$ de largeur avant de sortir de l'emprise.

L'ensemble 4 (F 6/105) mesure $32 \mathrm{~m}$ de longueur. Il joint l'ensemble 1 à l'ensemble 3 avec lequel il est perpendiculaire. Son profil est très irrégulier, avec des largeurs qui varient de 0,48 à $0,84 \mathrm{~m}$ et des profondeurs qui évoluent de 0,14 à $0,76 \mathrm{~m}$.

L'ensemble 5 (F 82, 49, 15) se compose de trois fossés que leurs orientations permettent d'associer pour former un enclos rectangulaire. Le fossé occidental mesure $32,50 \mathrm{~m}$, le côté sud $23 \mathrm{~m}$ et le côté oriental $10 \mathrm{~m}$. Leurs profils sont relativement variables; les largeurs évoluent de 0,90 à $1,40 \mathrm{~m}$ tandis que les profondeurs oscillent entre 0,50 


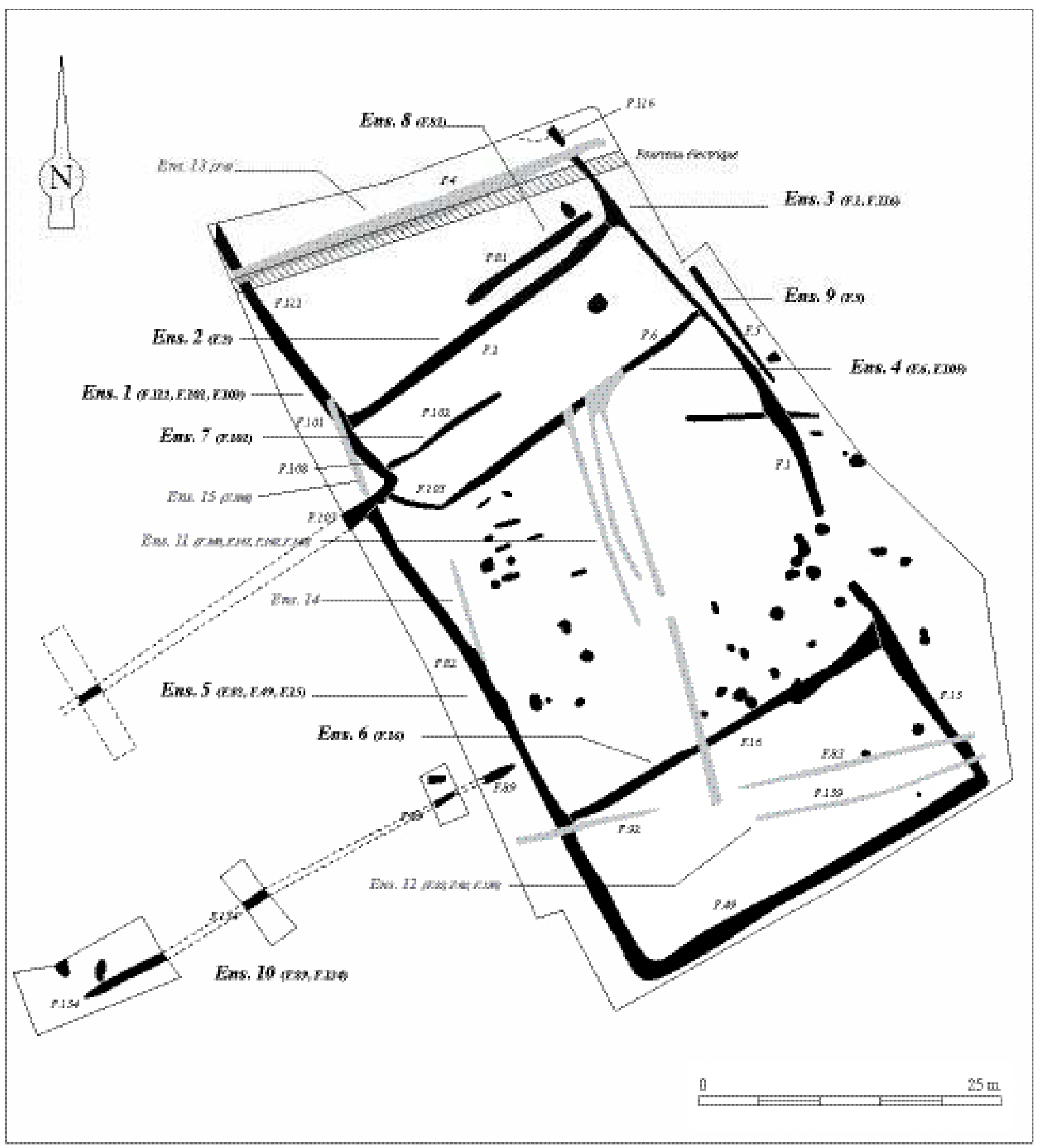

Figure 5 : Plan général des structures avec les différents ensembles.

Figure 5: General plan of features presenting the different structural sets.

et $0,74 \mathrm{~m}$. Au nord-est, le fossé F 15 , s'interrompt, face à l'arrêt de F 1 (ens. 3). Cette discontinuité d'environ $7 \mathrm{~m}$ correspond à une entrée.
L'ensemble 6 (F 16) est constitué d'un fossé rectiligne qui a été suivi sur $29,40 \mathrm{~m}$. Il est perpendiculaire aux fossés est et ouest de l'ensemble 5 qu'il vient ainsi compartimenter. 
Les largeurs observées varient de 0,90 à $1 \mathrm{~m}$ pour 0,30 à 0,50 de profondeur.

L'ensemble 7 (F 102) se compose d'une unique limite rectiligne de 11,20 m, perpendiculaire à l'ensemble 1. Ce fossé mesure de 0,55 à $0,70 \mathrm{~m}$ de largeur et de 0,25 à $0,38 \mathrm{~m}$ de profondeur.

L'ensemble 8 (F 81) est formé par un seul fossé parallèle à l'ensemble 2 . Il mesure $12,60 \mathrm{~m}$ de longueur, pour $0,70 \mathrm{~m}$ de largeur et des profondeurs comprises entre 0,25 et $0,45 \mathrm{~m}$.

L'ensemble 9 (F 5), d'un peu plus de $11 \mathrm{~m}$ de longueur, longe l'ensemble 3 . Il est mal conservé avec $0,50 \mathrm{~m}$ de largeur et jamais plus de $0,10 \mathrm{~m}$ de profondeur.

L'ensemble 10 et les fossés 134 et 89 qui le composent se retrouve dans plusieurs sondages sur plus de $42 \mathrm{~m}$ de longueur. Il est perpendiculaire à l'ensemble 5 et mesure une quinzaine de centimètres de profondeur pour des largeurs voisines de $0,70 \mathrm{~m}$.

\section{Les autres fossés: un réseau plus récent.}

Ces limites présentent des orientations différentes de celles des autres ensembles. L'ensemble 11 se compose de plusieurs fossés parallèles qui mesurent une trentaine de centimètres de largeur et $0,10 \mathrm{~m}$ de profondeur. Les fossés de l'ensemble 12, qui leur sont perpendiculaires, sont disposés en deux axes séparés par une bande de $5 \mathrm{~m}$ de largeur; celle-ci pourrait correspondre à un chemin. L'ensemble 13, au nord, se confond avec un fossé qui longe un chemin encore visible sur la photographie aérienne du début des années 90. L’ensemble 14, constitué d'un unique fossé de quelques mètres de longueur, recoupe nettement l'ensemble 5 et reprend l'orientation des ensembles 11 et 12 . Il en est de même pour le fossé F. 108 (ensemble 16).

\section{Étude sédimentaire des fossés}

Une quinzaine de relevés de coupes ont été réalisés à partir des sondages transverses aux fossés. Tous, à l'exception de F 2 (ensemble 2), présentent un remplissage comparable. Les sédiments observés, c'est à dire la partie restante du comblement non tronquée par les labours, présentent deux niveaux superposés :

- le niveau inférieur, est constitué de plaquettes de schiste en position non contrainte, c'est-à-dire suivant une inclinaison définie par le profil d'équilibre du remplissage;

- le second niveau est composé de plaquettes et de blocs de schiste et, plus rarement, de quartz, emballés dans une matrice limoneuse, le tout sans organisation perceptible.

Le niveau inférieur correspond à un remplissage naturel du fossé par accumulation de plaquettes de schiste détachées des parois. Le substratum (cf. supra) est de nature à fournir de telles plaquettes sous l'action du gel/dégel ou de toute autre action mécanique (piétinement). Le fossé F 2 (ensemble 2), fouillé par J.-C. Meuret durant l'évaluation de 1999, illustre notre propos : laissé ouvert pendant presque deux ans, le fond s'en est spontanément tapissé de plaquettes de schiste (fig. 6).

Le niveau supérieur a une composante sédimentaire graveleuse qui le distingue des sols limoneux environnants; le comblement des fossés ne s'est donc pas fait à leur détriment. Les plaquettes de schiste présentes dans ce comblement sont en position contrainte; de plus, il n'y a pas de dissymétrie du remplissage avec, côté du présumé talus, une composante sédimentaire plus graveleuse. Le remplissage de la structure, déconnecté des sources sédimentaires voisines (sol et talus) est donc le résultat d'un comblement volontaire et rapide.

Il est tentant de voir dans ce comblement final la matérialisation du démantèlement des super structures (probables talus) afin de niveler la parcelle. La reprise du fossé F 112, par le fossé F 108 (sondage F $112 \mathrm{~K}$ ) tend d'ailleurs à confirmer cette hypothèse. $\mathrm{F} 108$, postérieur à $\mathrm{F} 112$ et donc postérieur au nivellement supposé du terrain, est rempli de limons. La nature de son comblement se rapproche de celle des sédiments qui constituent les sols environnants et non pas de structures réalisées avec les déblais des fossés (talus par ex.).

\section{Une histoire d'enclos - Proposition de phasage}

Le site de Vauvert se compose d'une juxtaposition d'enclos. Un seul a été totalement décapé, les autres n’ont été que partiellement perçus parce qu'en grande partie situés hors du zonage archéologique établi avant l'opération. En

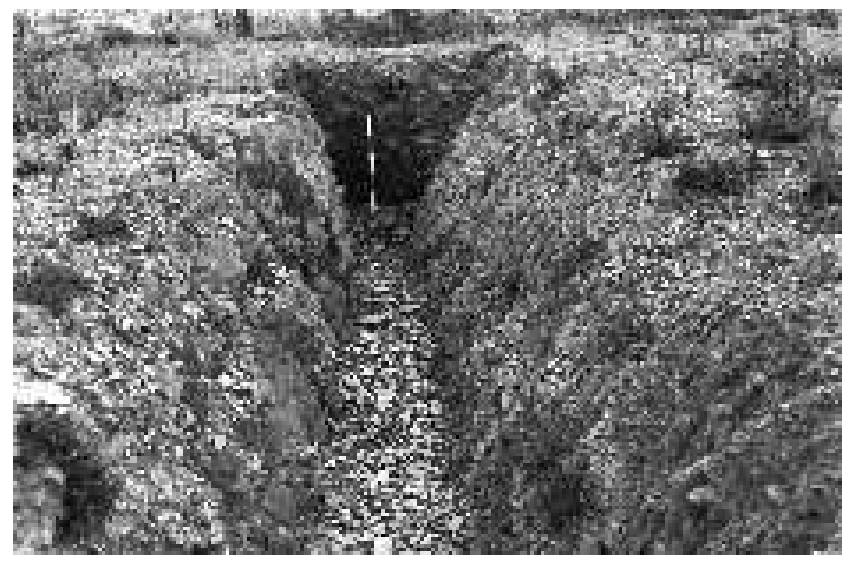

Figure 6 : Vue depuis l'ouest du fossé de l'ensemble 2 fouillé lors de l'opération de 1999 (état en 2001).

Figure 6: Ditch of set 2 seen from West (excavated in 1999; condition in 2001). 
s'appuyant sur les données spatiales, stratigraphiques et chronologiques, nous allons tenter de retracer l'évolution de ce site dont, nous n'avons malheureusement perçu que la périphérie (fig. 7).

Phase I - La partie la plus ancienne est en grande partie hors de l'emprise étudiée. Ce premier enclos (ensemble 1), sur lequel une grande partie des autres fossés (ensembles 2, 7,4 et 5) vient s'appuyer, est, de ce fait, le plus ancien. Le mobilier recueilli en remplissage indique une période comprise entre la fin du $\mathrm{VI}^{\mathrm{e}}$ siècle et la fin du siècle suivant. Perçu au travers de son seul angle est, il se développe vers le sudouest et le nord-ouest. Il mesure au moins $27 \mathrm{~m}$ du nord au sud et 32 à $33 \mathrm{~m}$ d'est en ouest si on lui associe le tronçon de fossé retrouvé dans un sondage extérieur au décapage. Aucune entrée n’a été repérée.

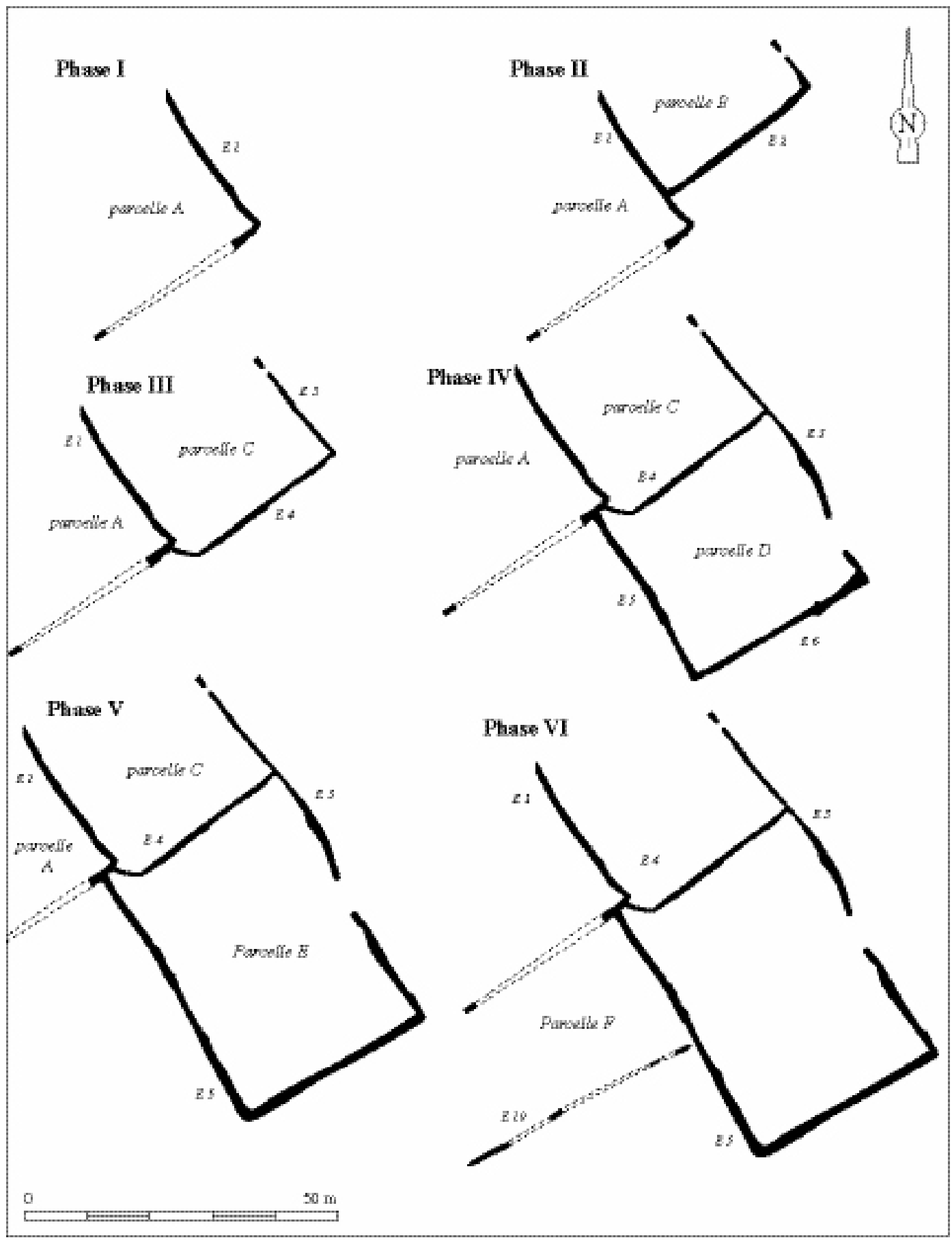

Figure 7 : Essai de phasage des enclos. Figure 7: Trial phasing of enclosures. 
Phase II - Plus tard, un second enclos (parcelle B) est établi avec l'ensemble 2 qui vient se greffer sur le côté nord-est de l'ensemble 1. À cette occasion, le fond du fossé 112 est recreusé (fig. 8). À l'est, ce deuxième enclos parait associé au fossé F 116, qui montre une interruption, ainsi qu'à l'extrémité septentrionale de l'ensemble 3. L'enclos serait alors ouvert de ce côté. La datation radiocarbone réalisée dans le remplissage de ce fossé au cours de l'évaluation de J.-C. Meuret, indique, nous l'avons évoqué, que nous nous situons entre le milieu du viI ${ }^{\mathrm{e}}$ siècle et le milieu du viII ${ }^{\mathrm{e}}$ siècle.
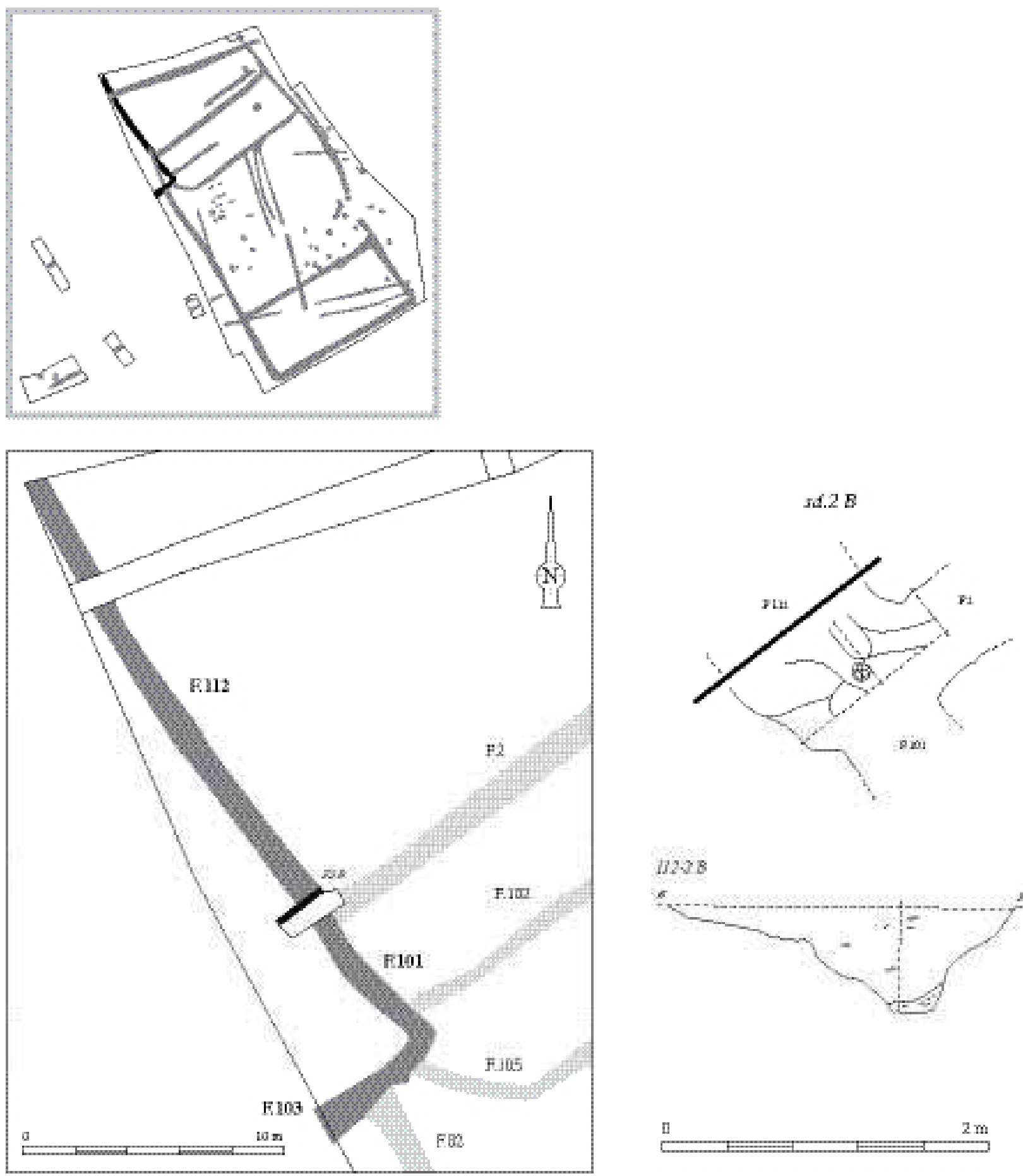

\section{$132 \sqrt{3}$}

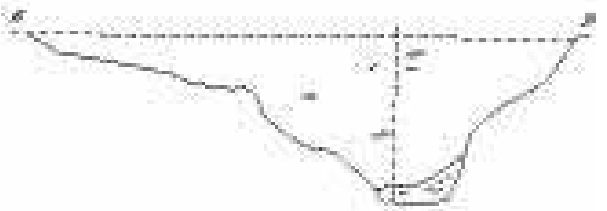

Figure 8 : Coupe stratigraphique du contact entre les ensembles 1 et 2. Figure 8: Stratigraphy of contact beween sets 1 and 2. 
Phase III - La parcelle B semble ensuite agrandie avec la création de l'ensemble 4 qui vient se greffer sur l'ensemble 1. Le relevé (fig. 9) montre que le premier (E 4) a fonctionné en même temps que le second avec une phase de remplissage commune aux deux fossés. Cette coupe montre également que l'ensemble 4 a semble-t-il été remblayé avant l'ensemble 1.

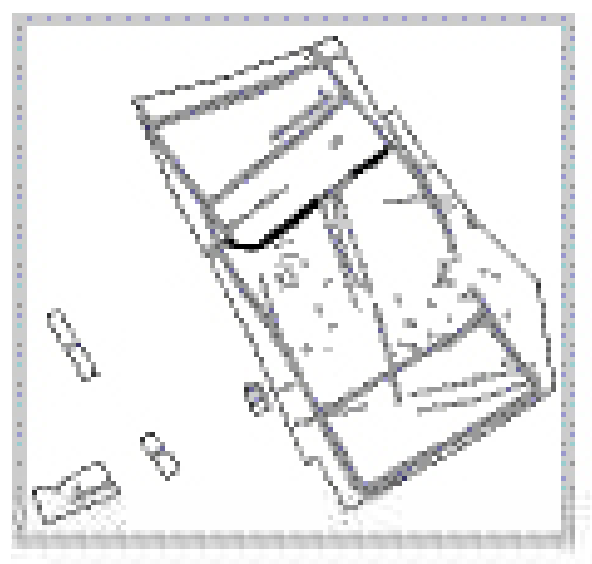

À l'est, en revanche, aucune relation stratigraphique n'a été observée entre les ensembles 3 et 4 . Ce nouvel enclos (parcelle C), dont la limite sud aurait ainsi été repoussée, devait toujours être ouvert à l'est.

Phase IV - Le site s'étend encore vers le sud-est. L'ensemble 5 et son fossé (F 82) en marquent le côté ouest; il vient se greffer sur l'ensemble 1. La nouvelle parcelle (D) est limitée au sud-est par l'ensemble 6 (F 16). À l'est, le côté est formé par F 115 et surtout par l'ensemble 3, qui semble alors

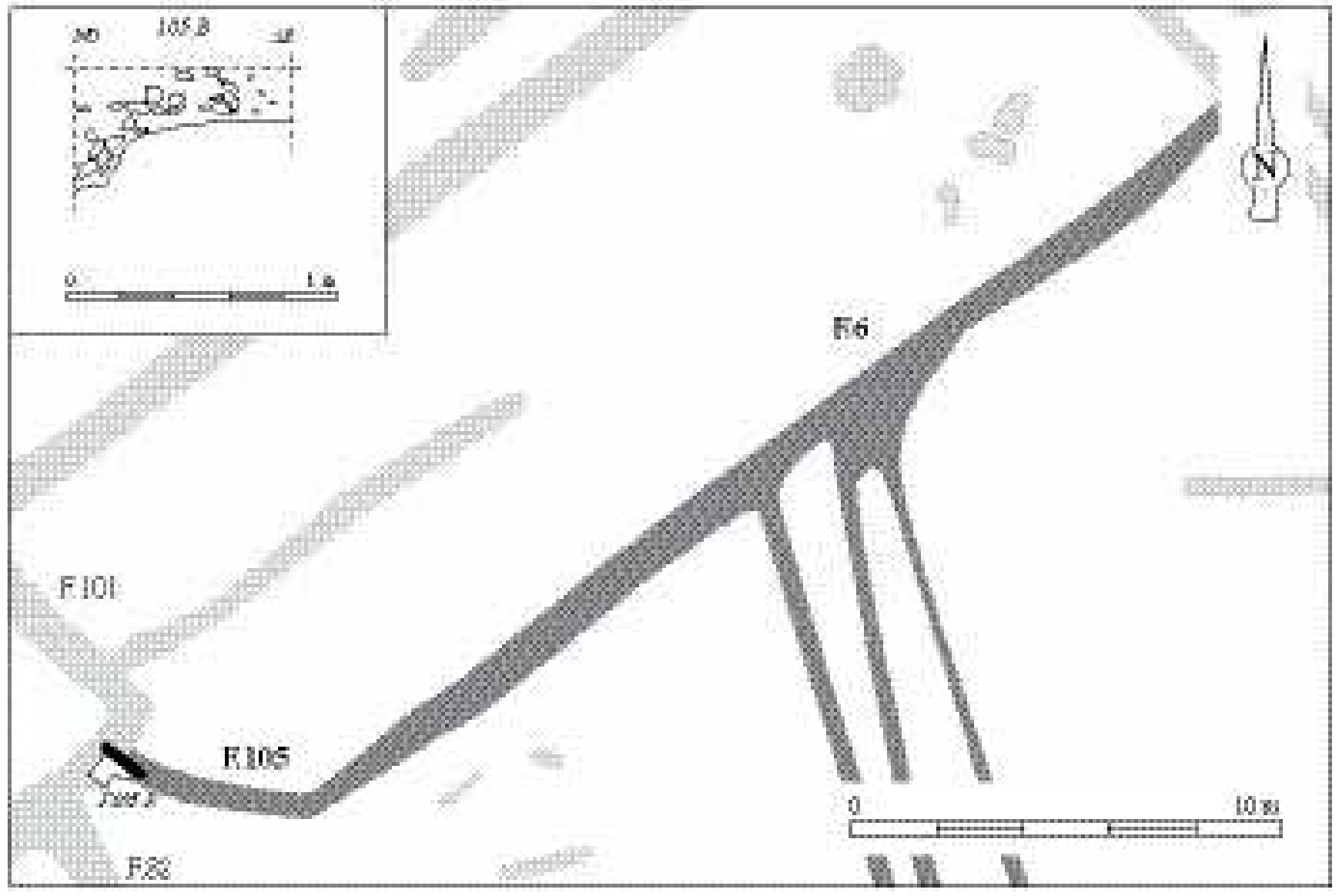

Figure 9 : Coupe stratigraphique relevée au contact des ensembles 1 et 4 .

Figure 9: Stratigraphy of contact between sets 1 and 4. 
Phase V-Elle voit encore une extension du site vers le sud. Une parcelle E plus vaste (environ $42 \mathrm{~m}$ sur 30) est cernée par les autres fossés de l'ensemble 5. À cette occasion, le fossé de l'ensemble 6 semble au moins en grande partie comblé comme l'indique la coupe 82A. Il apparait en effet que ce comblement intervient alors que l'ensemble 6 l'est déjà en grande partie (fig. 10). Dès lors, l'entrée est exactement au milieu du côté oriental de la parcelle $\mathrm{E}$.
Phase VI - L'ensemble 10 est créé à l'ouest. On ne sait pas si ce nouvel espace voit le jour immédiatement après la phase IV ou, pourquoi pas, plus tôt encore. Il est implanté contre le côté sud-est de l'enclos A, qui constitue toujours le cœur du site comme l'indiquent les tessons plus tardifs découverts dans le fossé F 101. Ce dernier reste ainsi l'un des axes structurant du gisement depuis la phase 1 jusqu'à la phase VI.

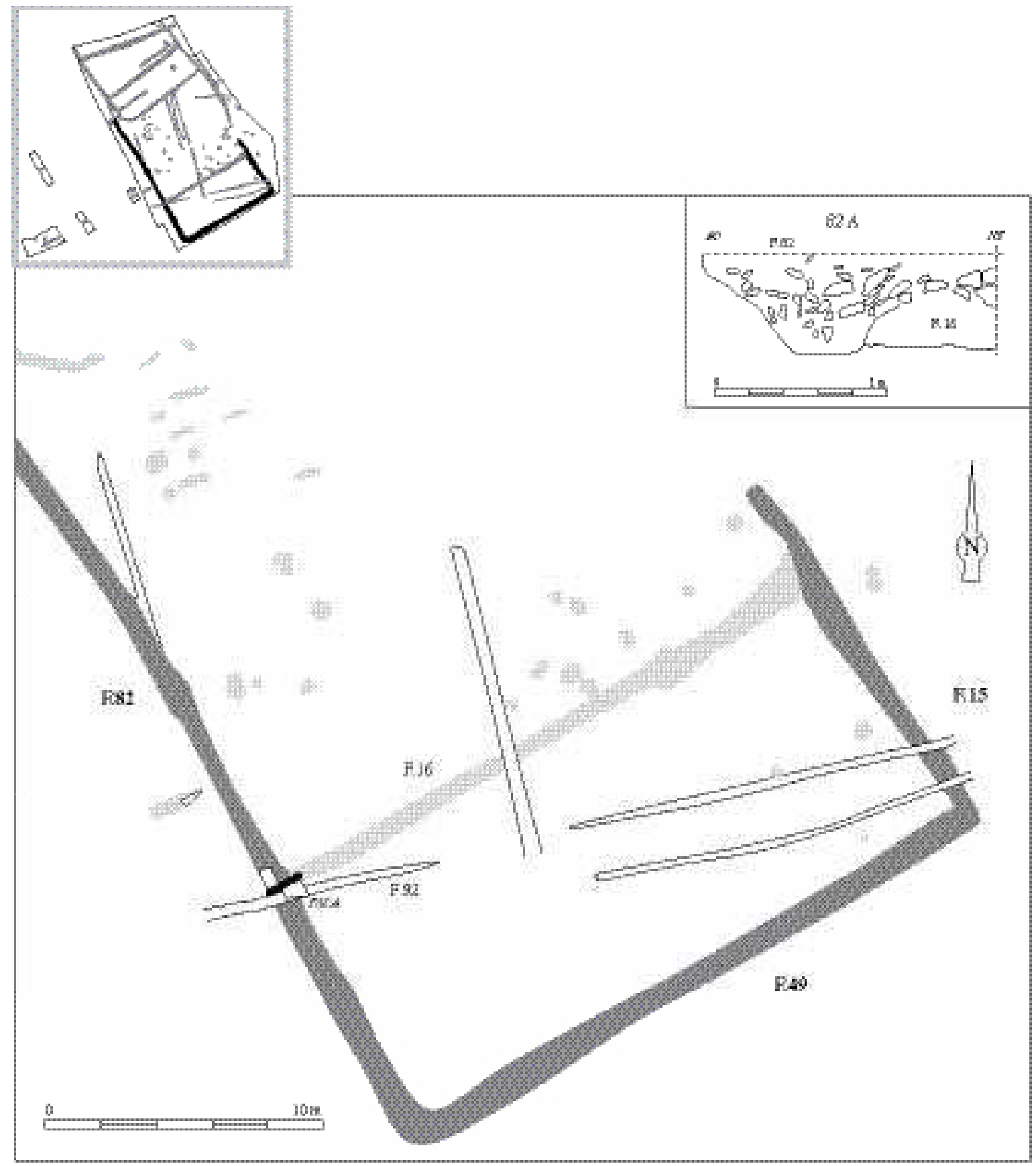

Figure 10 : Relations stratigraphiques entre les ensembles 5 et 6 .

Figure 10: Stratigraphic connections between sets 5 and 6 . 


\section{LES AUTRES STRUCTURES}

\section{Des trous de poteaux et des anomalies}

\section{La concentration sud-est}

Les creusements pouvant évoquer des trous de poteaux sont concentrés essentiellement aux abords de l'entrée de la parcelle D/E (fig. 5 et 11). Sur la trentaine de structures étudiées, huit seulement ont été identifiées, d'après leur profondeur (au moins 0,16 m conservés) et leur profil, comme de véritables trous de poteaux. Onze autres (entre 0,10 et $0,15 \mathrm{~m}$ de profondeur) ont posé d'avantage de problème d'interprétation. Pour les dernières (entre 0,04 et 0,09 m), leur mauvaise conservation n'a pas permis de trancher entre chablis, dépressions liées à l'érosion ou faits d'origine anthropique.

Toutefois, une petite concentration de trous de poteaux avérés existe dans l'axe de l'entrée de l'enclos. Certains comme $\mathrm{F} 13$ et $\mathrm{F}$ 51, de fort diamètre (au moins $1,50 \mathrm{~m}$ ) et de profondeur importante $(0,54 \mathrm{~m})$, pourraient être liés à un dispositif de fermeture. L'espace séparant les extrémités des fossés et ces trous aurait alors été occupé par l'extrémité des talus. Il faut toutefois souligner que l'absence de mobilier dans ces creusements incite à la prudence. On ne peut même pas être sûr que tous appartiennent bien au haut Moyen Âge...

Pour le reste des structures, elles sont souvent trop arasées et leur répartition beaucoup trop aléatoire pour qu'y soit décelable la moindre construction.

\section{La concentration ouest}

Une seconde concentration a été relevée à l'ouest de la parcelle D (fig. 12). Elle ne rassemble qu'une quinzaine de creusements ou assimilés. Parmi eux, presque la moitié, beaucoup trop arasée est à exclure du débat (F 91, 87, 126 et probablement $88,93,109$ et 123$)$. On note en revanche, deux trous de poteaux évidents : F 96, qui présente des éléments de calage, et F 122, avec un plan circulaire et une profondeur de $0,20 \mathrm{~m}$.

D'autres creusements bien différents, au nombre de six, ont attiré notre attention. Il s'agit de fosses oblongues dont les longueurs vont de 127 à $220 \mathrm{~cm}$ pour 8 à $34 \mathrm{~cm}$ de largeur et 7 à $24 \mathrm{~cm}$ de profondeur. Les remplissages n'apportent pas d'éléments particuliers. Excepté F 124 et 119, toutes ces fosses ont une orientation globalement est-ouest. Seule F 124 a une orientation qui se rapproche de celle du fossé $F$ 6. Cinq de ces structures sont regroupées, la sixième (F 131) étant un peu à l'écart. Les profils sont réguliers, excepté $\mathrm{F} 121$ qui montre un léger surcreusement dans sa partie occidentale. Aucune de ces fosses n'a livré de mobilier mais l'absence quasi-générale sur le site d'autres périodes que le haut Moyen Âge est un élément qui permet de les rattacher à cette phase chronologique. Aucune interprétation ne prévaut pour ces fosses si ce n'est qu'elles sont semblables à celles découvertes sur le site de La Tène finale voisin du Clos-Henry où quatre structures signalent la présence d'un petit bâtiment carré de type grenier. Plusieurs de ces anomalies avaient déjà été relevées lors de l'évaluation de Vauvert par J.-C. Meuret (fig. 4; Meuret, 1999, p. 7). L'hypothèse de tronçons de sablières basses avait alors été proposée. Malheureusement, il est bien difficile de trancher car il manque en particulier les structures perpendiculaires qui pourraient compléter les plans d'éventuels bâtiments.

\section{Un four}

La fosse $\mathrm{F} 9$ a été relevée à proximité de l'ensemble 8 (F 81). En plan, le creusement de forme sub- rectangulaire mesure 1,5 m de longueur sur 0,84 $\mathrm{m}$ de largeur maximale. La structure, orientée Nord-Nord-Ouest/Sud-Sud-Est, est dotée d'une partie plus large et régulière (moitié sud-est). À l'opposé, elle est plus étroite et s'achève même en pointe (fig. 13).

Ce creusement atteint $0,19 \mathrm{~m}$ de profondeur dans sa partie la plus large. Vers l'extrémité nord-ouest en revanche, le fond remonte en pente régulière jusqu'à atteindre le niveau du décapage. Le remplissage se compose d'une seule phase mêlant du limon brun moyen à des plaquettes de schiste. Il n'a livré aucun mobilier. Cette fosse peu profonde montre d'évidentes traces de rubéfaction mais uniquement sur les deux tiers les plus larges de la structure.

La forme générale de ce creusement, son profil et surtout ses traces de rubéfaction permettent de l'assimiler à un four. Par comparaison avec des exemplaires mieux conservés, il est possible, d'après son profil et son plan, de l'orienter : sa bouche se situe assurément du côté le plus étroit, la partie large et rubéfiée constituant la chambre de chauffe. Le four de Vauvert peut être comparé à un exemplaire carolingien du site de la Frétellière à Trémentines (Maine-etLoire : Dubillot et Valais, 2006, p. 56); il s'agit d'un four à chambre unique dont l'entrée se situe du côté le plus étroit. On peut probablement restituer à Vauvert une structure de combustion entièrement excavée d'une cinquantaine de centimètres de profondeur (par rapport au niveau de la parcelle actuelle). Elle était sans doute accessible depuis une fosse située devant la gueule. Dans ce cas, et faute de rejets spécifiques de type " tessonnière " comme à la Frétellière, il est difficile d'en interpréter la fonction exacte mais il est probable que l'exemplaire de Vauvert n'ait rempli qu'une fonction domestique. Malgré l'absence de mobilier issu de son remplissage, nous proposons d'attribuer cette structure de combustion à la période d'occupation du site qui couvre 

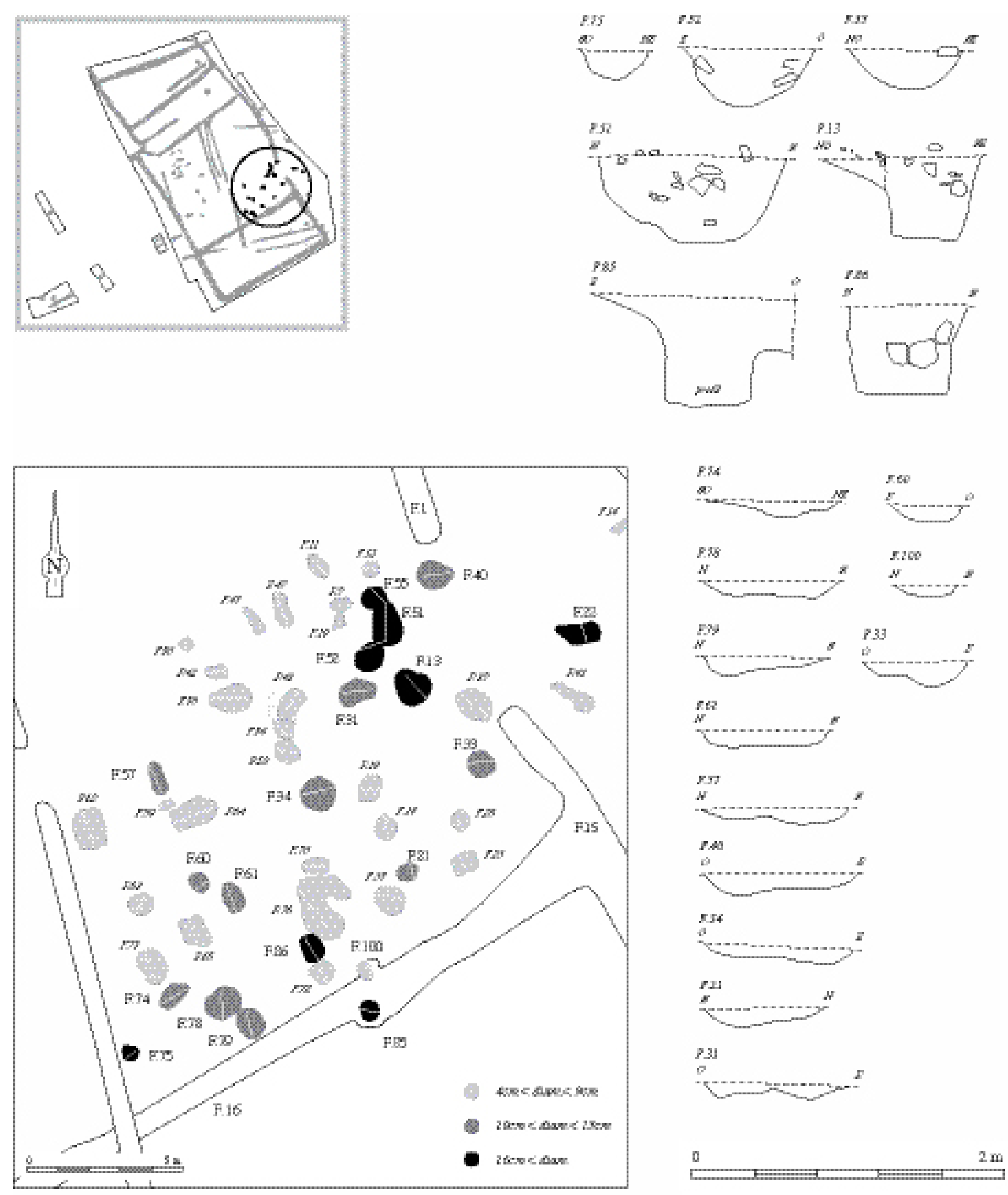

Figure 11 : La zone de concentration de structures à proximité de l'entrée de la parcelle $\mathrm{D}$ (les traits blancs sur le plan indiquent l'emplacement des coupes).

Figure 11: Concentration of features near the entrance of plot D. (white lines o the plan mark the sections). 

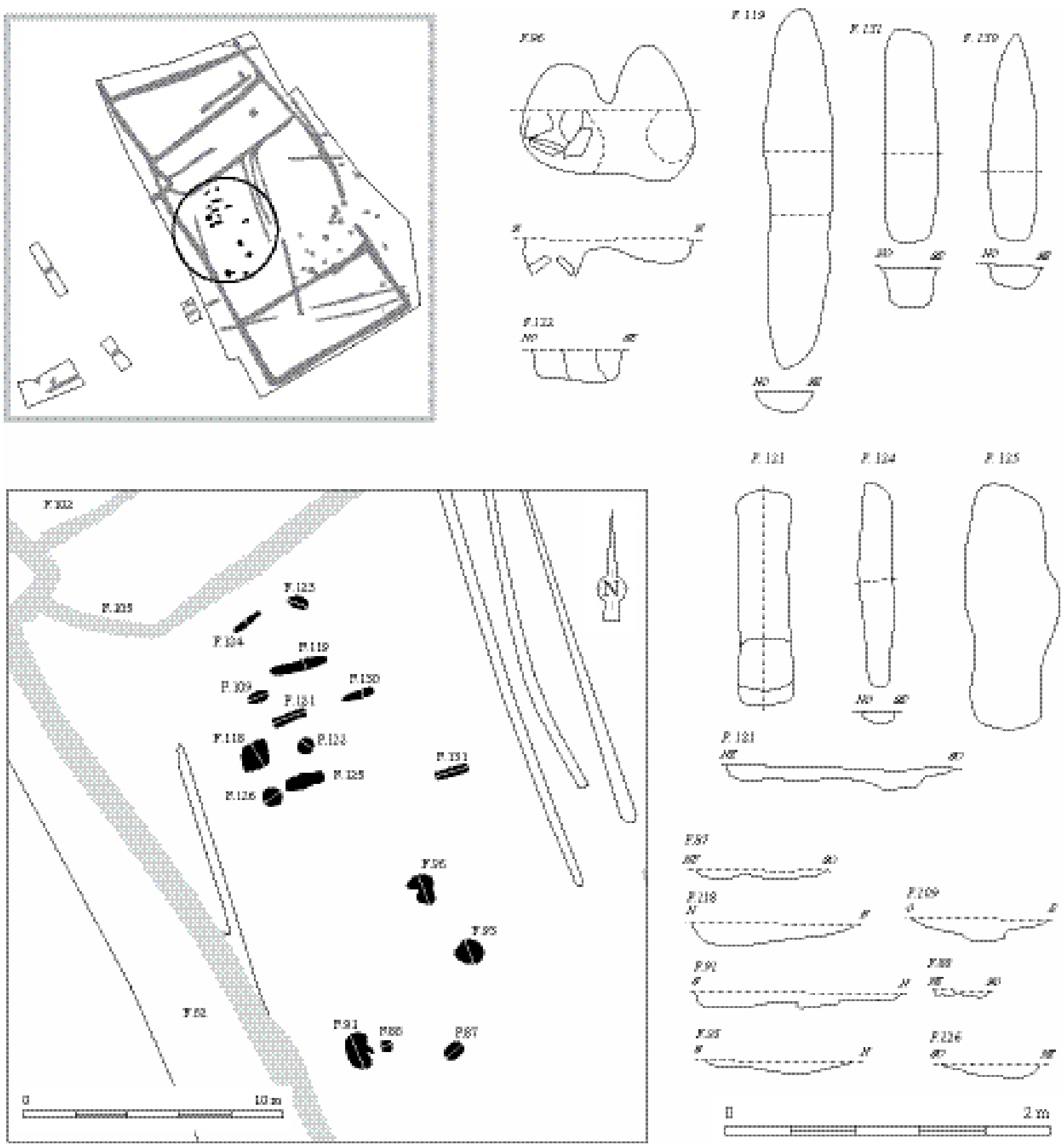

Figure 12 : Une seconde zone de concentration de structures dans la partie ouest de la parcelle D.

Figure 12: Second concentration of features in western part of plot D. 

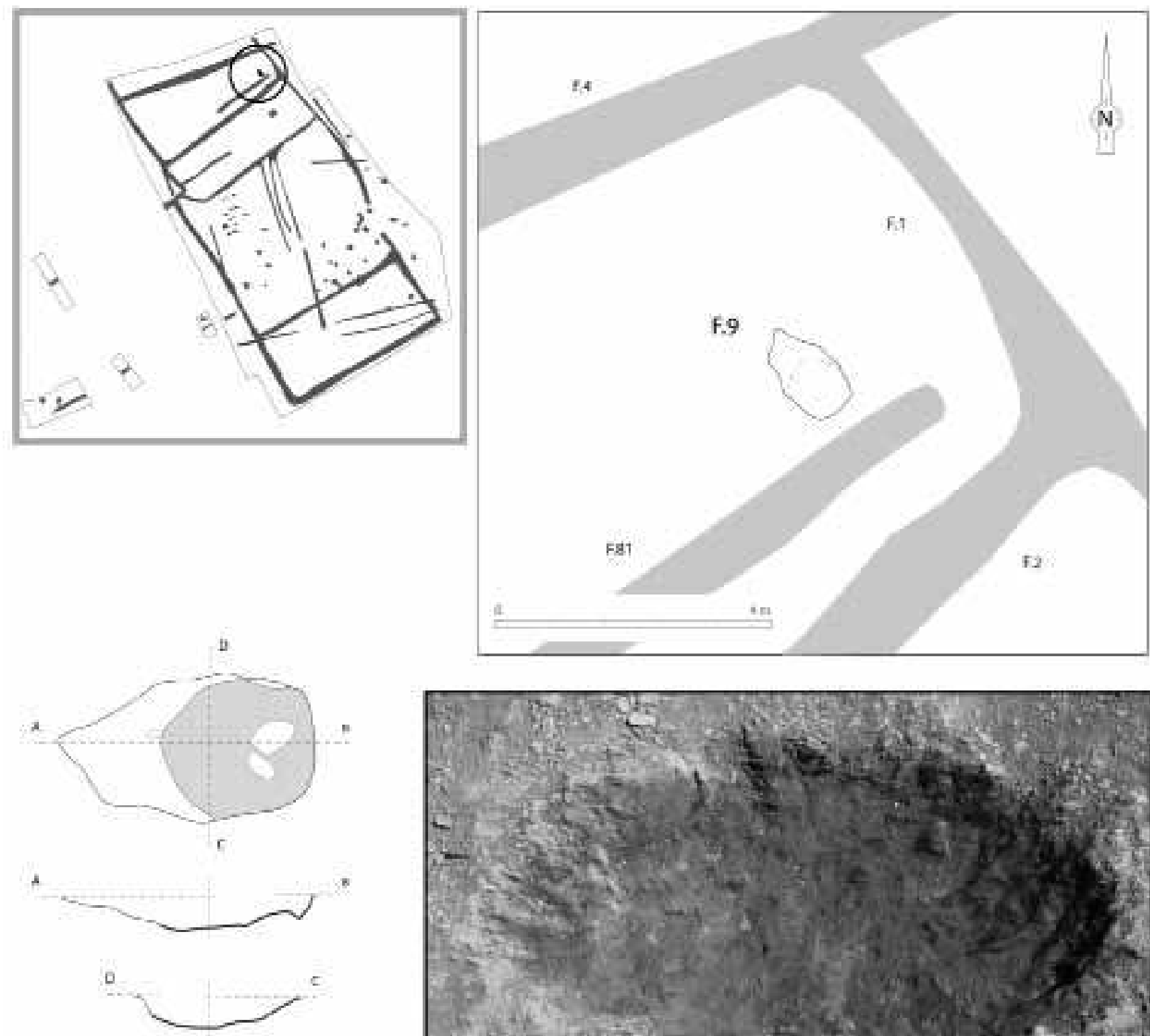

Tone nheste

0.

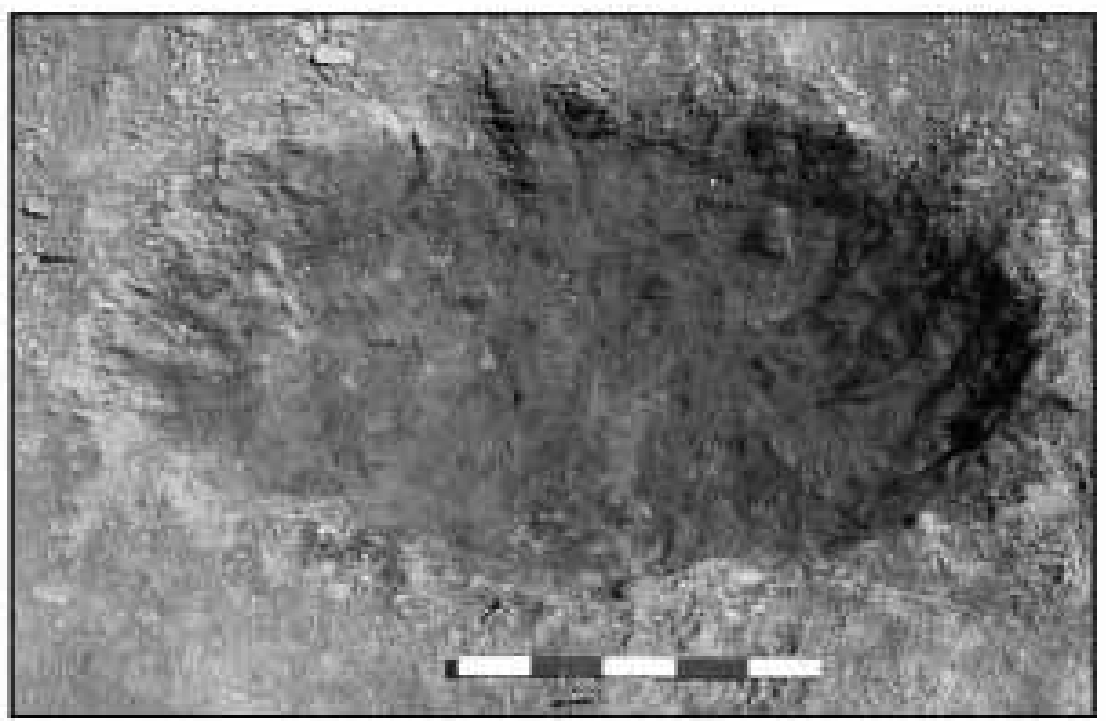

Figure 13 : Relevés et photographie du four F 9.

Figure 13: Plan, sections and photograph of kiln F9.

la fin de la période mérovingienne et le début de la période carolingienne ( $\mathrm{VII}^{\mathrm{e}} / \mathrm{VIII}^{\mathrm{e}}$ siècles).

\section{Une fosse à quatre trous de poteaux}

La fosse $\mathrm{F} 3$, de forme rectangulaire, mesure 1,60 sur 1,40 m. Située entre l'ensemble 4 (F 6) et l'ensemble 2 (F 2), elle est orientée sud-ouest/nord-est (fig. 14). Son plan est régulier si ce n'est du côté sud-sud-ouest qui présente une légère avancée en son centre; cette partie est aussi légèrement plus étroite. D'abord fouillé par moitié, ce creusement atteint $0,70 \mathrm{~m}$ de profondeur, dimension qui nous a conduits à le dégager en totalité. Cette fosse présente une morphologie régulière et des parois abruptes. Le fond est composé de deux parties rectangulaires. 

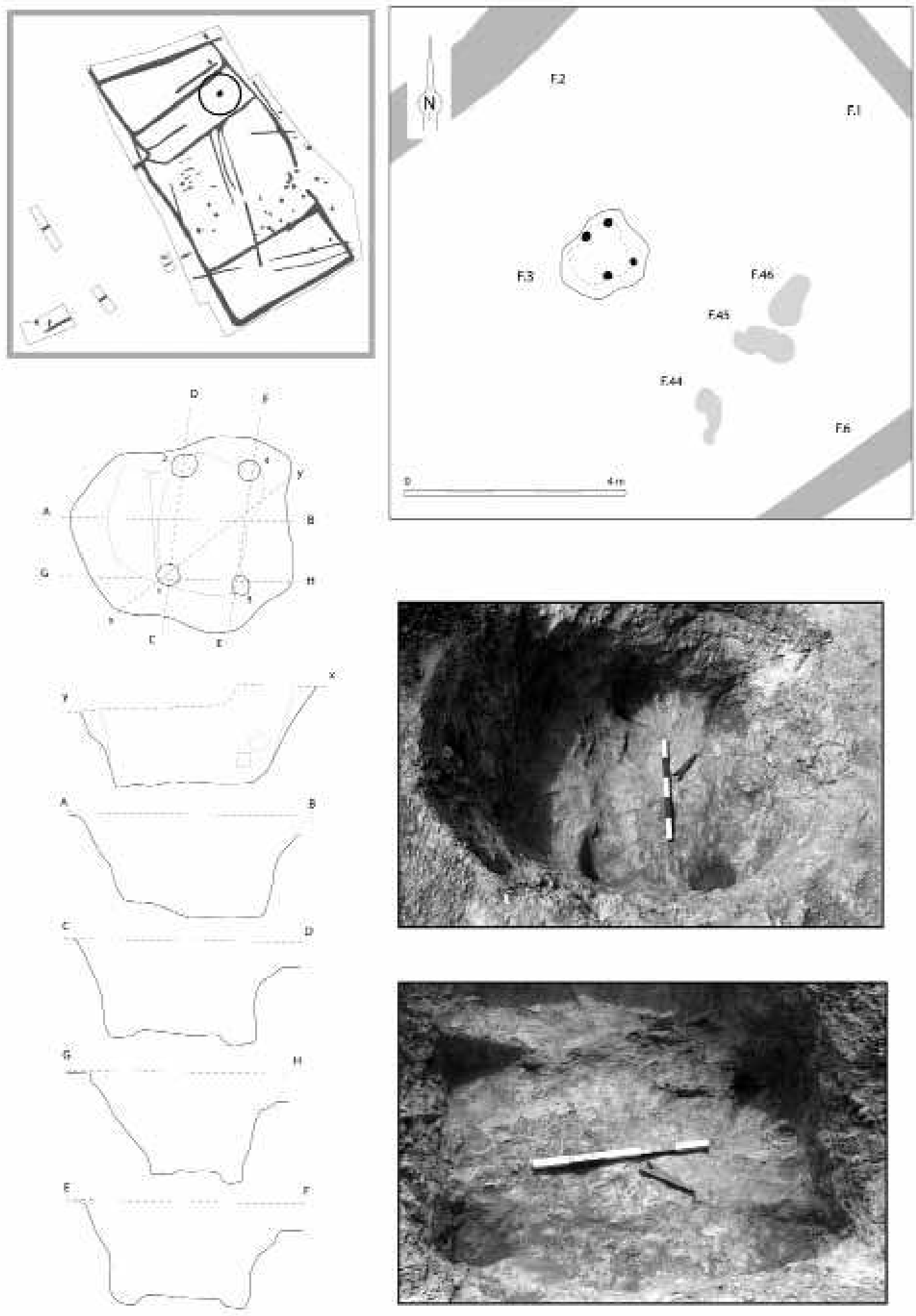

0

$2 m$

Figure 14 : Relevés et photographies de la fosse à quatre poteaux $\mathrm{F} 3$.

Figure 14: Plans, sections and photographs of four-posted pit F3. 
- La première, qui appartient à la partie la plus large de la fosse, en constitue également la zone la plus profonde. Avec un fond parfaitement plan, elle mesure $0,60 \mathrm{~m}$ de largeur pour $1 \mathrm{~m}$ de longueur. De plus, elle possède l'empreinte d'un poteau à chacun de ses angles.

- La seconde, de même orientation, appartient à la partie la plus étroite du creusement et domine d'une dizaine de centimètres la partie dotée de trous de poteau. D'un profil régulier, cette zone mesure de 0,24 à $0,30 \mathrm{~m}$ de largeur pour $0,80 \mathrm{~m}$ de longueur. Le remplissage ne livre pas d'informations particulières en dehors de quelques tessons du haut Moyen Âge.

L'empreinte de quatre poteaux dans le fond de cette fosse marque sans doute les points d'assise d'un bâti, d'un cuvelage ou même d'une toiture. La partie rectangulaire plus étroite ressemble à une marche qui permettrait de descendre dans la fosse depuis son côté le plus étroit. S'agit-il d'une minuscule construction excavée destinée au stockage, à une activité artisanale ou encore à des latrines? Rein ne permet de trancher.

Dans la région, une fosse découverte dans la basse-cour d'une motte féodale à Courgains rappelle bien les dispositions générales de cette découverte. Dotée également de quatre poteaux, elle mesure 2,20 x 1,06 m mais seulement $0,50 \mathrm{~m}$ de profondeur. Attribuée au XIII ${ }^{\mathrm{e}}$ siècle, aucune interprétation ne prévaut non plus dans son cas (Valais et Dumas, 2006, p. 116-117). Ailleurs, en Île-de-France par exemple, les sites de Saint-Pierre-du-Péray et de Saint-Germain-lesCorbeil (Essonne) en ont livré également (communication orale F. Gentili). On a parfois avancé l'hypothèse de pressoirs... (Chapelot, 1993, p. 190).

D'autres découvertes comparables à cette fosse à quatre poteaux ont également été réalisées à proximité immédiate de la basilique de Saint-Denis (Meyer, 1993). Il s'agit ici de quatre creusements conservés sur des profondeurs allant de 0,80 à $1,30 \mathrm{~m}$. Chacune de ces fosses carolingiennes était également dotée de creusements à chaque angle, pouvant mesurer jusqu'à $0,27 \mathrm{~m}$ de profondeur. La fouille minutieuse de l'une d'elle a permis d'identifier l'empreinte d'un coffre en bois d'au moins $0,87 \mathrm{~m}$ de hauteur et $0,72 \mathrm{~m}$ de largeur pour 1,37 $\mathrm{m}$ de longueur. Une serrure en fer était associée à cet ensemble. Le coffre était doté de quatre montants dont les bases reposaient dans les avant-trous réalisés aux angles de la fosse. À la lumière de cette découverte, et même si l'exemplaire de Vauvert n'est pas aussi régulier, il est donc possible d'envisager que ce creusement énigmatique y ait reçu également un coffre en bois dont la fonction exacte reste malheureusement inconnue.

\section{Une sépulture?}

Si le fossé F. 16 peut être clairement attribué au haut Moyen Âge, rien dans le remplissage de la sépulture présumée ne permet d'être aussi affirmatif. On peut raisonnablement admettre que cet aménagement, dont il ne reste malheureusement aucun élément organique, remonte à une période où le fossé était encore visible, son installation en ayant repris l'orientation. Notons également que la rareté des éléments d'occupation postérieure au premier millénaire sur le site milite elle aussi pour une datation du haut Moyen Âge. Il est donc probable que cette structure, probablement la tombe d'un jeune enfant au vu de ses dimensions, ait été aménagée alors que le fossé était déjà en partie rebouché, mais avant que le site ne soit totalement abandonné. Elle pourrait donc appartenir à l'ultime occupation de Vauvert et être contemporaine de la dernière phase d'enclos, à une période où $\mathrm{F} 49$ constitue le fossé le plus méridional du site, au viII siècle.

Plus largement, il est habituel de rencontrer pour cette période des sépultures isolées au sein des habitats ou à leur périphérie immédiate. La présence d'enfants dans ces contextes n'est d'ailleurs pas rare : il en existe plusieurs à Villiers-le$\operatorname{Sec}$ (Val d'Oise) où une sépulture de jeune enfant était située entre deux fours domestiques et une seconde également dans un fossé (Guadagnin, 1988, p. 170a). En Pays de la Loire, nous ne connaissons actuellement qu'un seul exemple de sépulture comparable, celle d'une jeune adulte installée dans le remplissage d'un fossé du site des Cinq-Chemins à Bauné (Maine-et-Loire), dans un contexte légèrement plus récent (autour du XI ${ }^{\mathrm{e}}$ siècle : David et Valais, 2003, p. 72).

Sur les sites du haut Moyen Âge, il n'est donc pas exceptionnel de rencontrer ce type de sépulture isolée. Dans la moitié nord de la France, ces découvertes deviennent fréquentes à partir de la seconde moitié du viI ${ }^{\mathrm{e}}$ siècle et durant tout le viII (Peytremann, 2003, p. 314). Les raisons de leur présence sont mal connues. On avance tantôt des raisons sociales ou culturelles qui auraient abouti à ces phénomènes de relégation (Guadagnin, 1988, p. 170), tantôt des raisons liées à la faiblesse de la contrainte paroissiale ou encore à la dispersion des habitats. Ces populations rurales pouvaient encore inhumer leurs morts sur leur terre (Garnotel et Raynaud, 1996, 147).

À l'occasion d'un sondage dans le fossé F 16, une probable sépulture construite a été découverte (fig. 15). Faute d'interprétation plus pertinente, nous avons en effet privilégiée cette hypothèse malgré une absence totale de restes osseux. De forme légèrement trapézoïdale, l'aménagement mesure $0,70 \mathrm{~m}$ de longueur sur 0,50 de largeur maximale. Il est établi au fond du fossé, élargi pour l'occasion. La partie la plus large se trouve au sud-ouest. Les plaques de schiste 

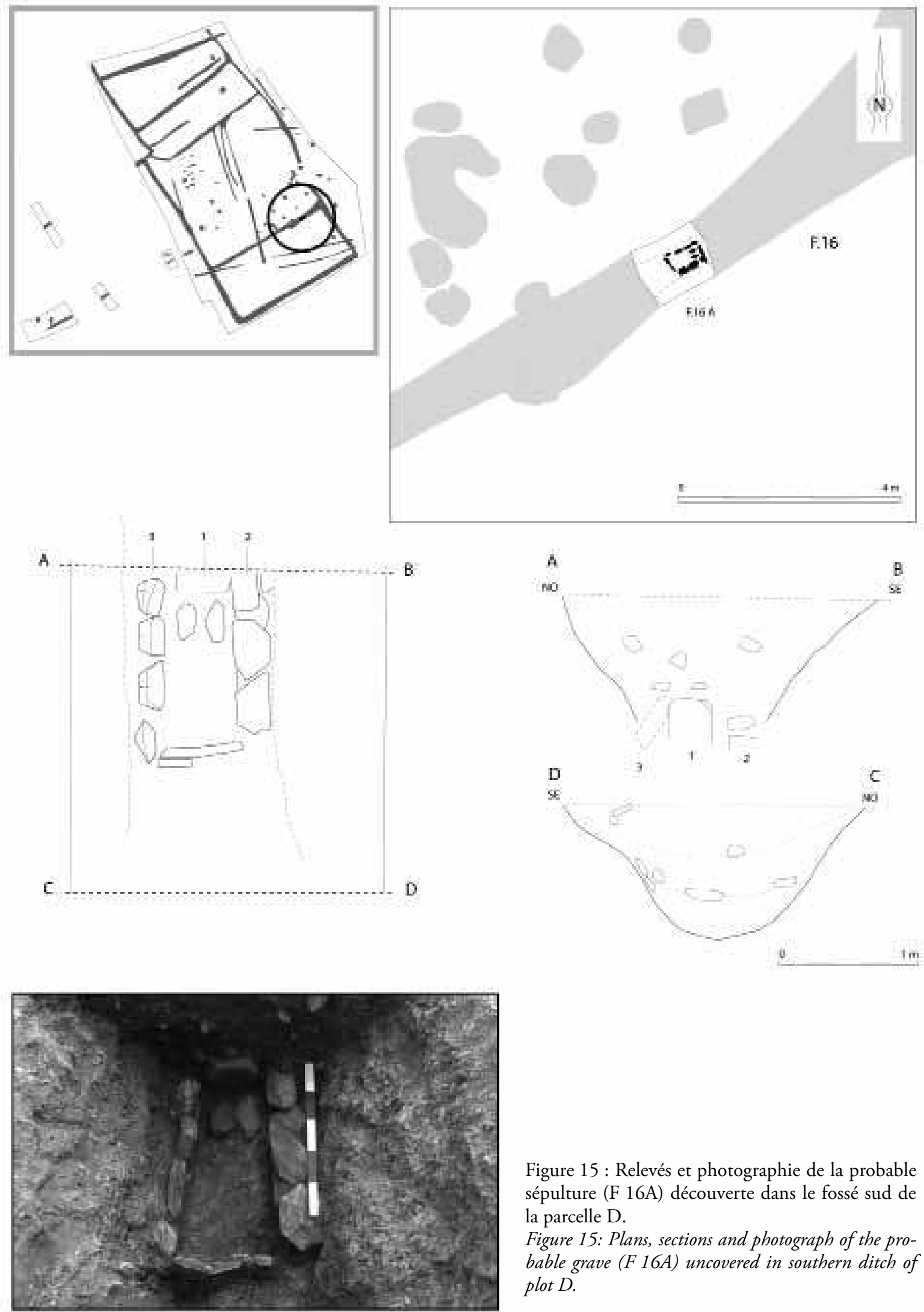

Figure 15 : Relevés et photographie de la probable sépulture (F 16A) découverte dans le fossé sud de la parcelle $\mathrm{D}$.

Figure 15: Plans, sections and photograph of the probable grave (F 16A) uncovered in southern ditch of plot D. 
qui composent les parois sont posées sur chant du côté nord; presque toutes ont basculé à l'intérieur. Au sud, les éléments de schiste sont empilés à plat sur deux assises. La partie de la «tombe " située au sud-ouest-ouest est constituée d'une plaque posée sur chant, détail qui existe également à l'extrémité opposée. Deux plaquettes de schiste ont été déposées à plat dans cette partie nord-est de l'aménagement.

\section{LE MOBILIER ARCHÉOLOGIQUE ET LES ÉLÉMENTS DE DATATION}

\section{La poterie}

\section{Présentation typologique}

Le matériel céramique recueilli est modeste puisqu'il ne se compose que d'une centaine de fragments (fig. 16). Nous le présenterons sommairement à partir de l'étude d'I. MoréraVinçotte. D'après les pâtes, les lèvres et les décors, la très grande majorité appartient au haut Moyen Âge. Seul un lot découvert dans la phase de remplissage terminal du fossé F. 82c appartient au bas Moyen Âge.

Pour le haut Moyen Âge, les pâtes sont le plus souvent grises assez claires, beiges et plus rarement brunes. Des traces plus sombres apparaissent parfois, sur des récipients approchés des flammes lors de leur utilisation. La céramique est bien cuite, avec un dégraissant sableux où les grains de quartz sont nombreux. L'aspect évoque tout à fait le type de poterie trouvé en Anjou, notamment sur les sites du Château d'Angers, des Cinq-Chemins à Bauné ou encore des Murailles à Distré, où les surfaces des céramiques sont également rugueuses (Moréra-Vinçotte, 2000, p. 60). L'ensemble du lot est constitué de vases tournés et décollés $\mathrm{du}$ tour à la ficelle. Un exemplaire montre une perforation juste au-dessus de son épaulement (fig. 16, F 112, sond. K, $\mathrm{n}^{\circ} 2$ ). De par sa position, il ne peut s'agir que d'un trou de réparation.

Les profils. Presque tous les individus étudiables sont des formes fermées, essentiellement des vases culinaires ou de stockage sans anses. Un seul récipient présente un profil semi-fermé (fig. 16, F 91). Il s'agit d'un vase à collerette, qui a pu être doté d'une anse. Les profils sont essentiellement ovoïdes. Plusieurs vases présentent un bourrelet qui en souligne l'épaulement (F 112, sond. K, n ${ }^{\circ} 1$ et 2). Un autre fragment montre un départ d'anse ou un bourrelet de préhension (F 101, sond. A, $\mathrm{n}^{\circ}$ 2).

Les décors. Confirmant ce qui avait été vu lors de l'évaluation, les seuls décors relevés sont tous réalisés à la molette. Il s'agit souvent de simples registres de casiers rectangulaires (F 2, sond. B), de carrés (F 91) ou de losanges (F 101, sond. $A, n^{\circ} 1$ ), situés au niveau de l'épaulement. Quand ce dernier est marqué par un bourrelet, le décor se situe juste au-dessous. Pour les deux premiers exemples, le registre se compose d'une superposition de deux lignes. Quelques tessons montrent des décors plus complexes : un exemplaire est constitué d'un registre d'oves ou d'arcades (F 112, sond. $\mathrm{K}, \mathrm{n}^{\circ} 1$ ), un autre montre deux lignes où alternent carrés et couples de petits rectangles (F 82, sond. C).

Les lèvres. Elles sont le plus souvent simples et fortement éversées (fig. 16, F 2, sond. B, $\mathrm{n}^{\circ} 1$ ). Un vase montre, un bord à collerette (F 91), un autre (F 101, sond. A, $\left.n^{\circ} 2\right)$ dispose peut-être, nous l'avons vu, d'un bourrelet de préhension retombant et légèrement concave. Sa face supérieure est creusée d'une profonde gouttière.

\section{La datation}

Les éléments céramiques les plus anciens sont les formes munies d'un bourrelet. Il s'agit des vases $\mathrm{F} 112 \mathrm{k}, \mathrm{n}^{\circ} 1$ et 2 (fig. 16). Ce type est courant de la fin du vie siècle au VII ${ }^{e}$. Dans la région, on en connaît un lot aux Cinq-Chemins à Bauné (David et Valais, 2003, p. 83) et un autre à la Sicardière à Saint-Georges-du-Bois (Pétorin et al., 1995). La forme semi-ouverte à collerette peut leur être associée; on en connaît notamment un exemplaire avec anse aux CinqChemins, et un autre sur le site de Senneçay à Mazé (MoréraVinçotte, 2000, pl. 2 et 3). Il semble que le reste du mobilier céramique soit légèrement plus récent (viII ${ }^{\mathrm{e}}$ siècle?), avec la disparition des bourrelets et une relative simplification dans le type de molette employée. Soulignons que cette tendance doit être utilisée avec prudence, le lot céramique étant ici fort restreint. Une forme toutefois est plus tardive : la lèvre en bandeau (F 101A n ${ }^{\circ}$ 2, fig. 16) appartient clairement à la période carolingienne voire même la fin de celle-ci.

\section{Autres éléments mobiliers}

\section{Disques céramiques}

Trois fonds de poteries ont été retaillés pour obtenir des disques réguliers, de 8 à $8,5 \mathrm{~cm}$ de diamètre pour les deux exemplaires mesurables. La fonction exacte de ces rondelles de céramique n'est pas établie pour l'instant faute de recherches bibliographiques approfondies. Le site de Rougiers en a fourni de nombreux exemplaires (environ 400), dans des contextes plus tardifs (xiv siècle : Démians d'Archimbaud, 1980, p. 418-421). Comme à Rougiers, la fonction de couvercle peut être écartée, ceux-ci étant bien connus pour le haut Moyen Âge, même sur les officines les plus modestes comme celles de la Frétellière (Dubillot et Valais, 2006, p. 62). L'hypothèse de jetons ou de palets de jeux peut être avancée, mais rien ne permet de la vérifier. 


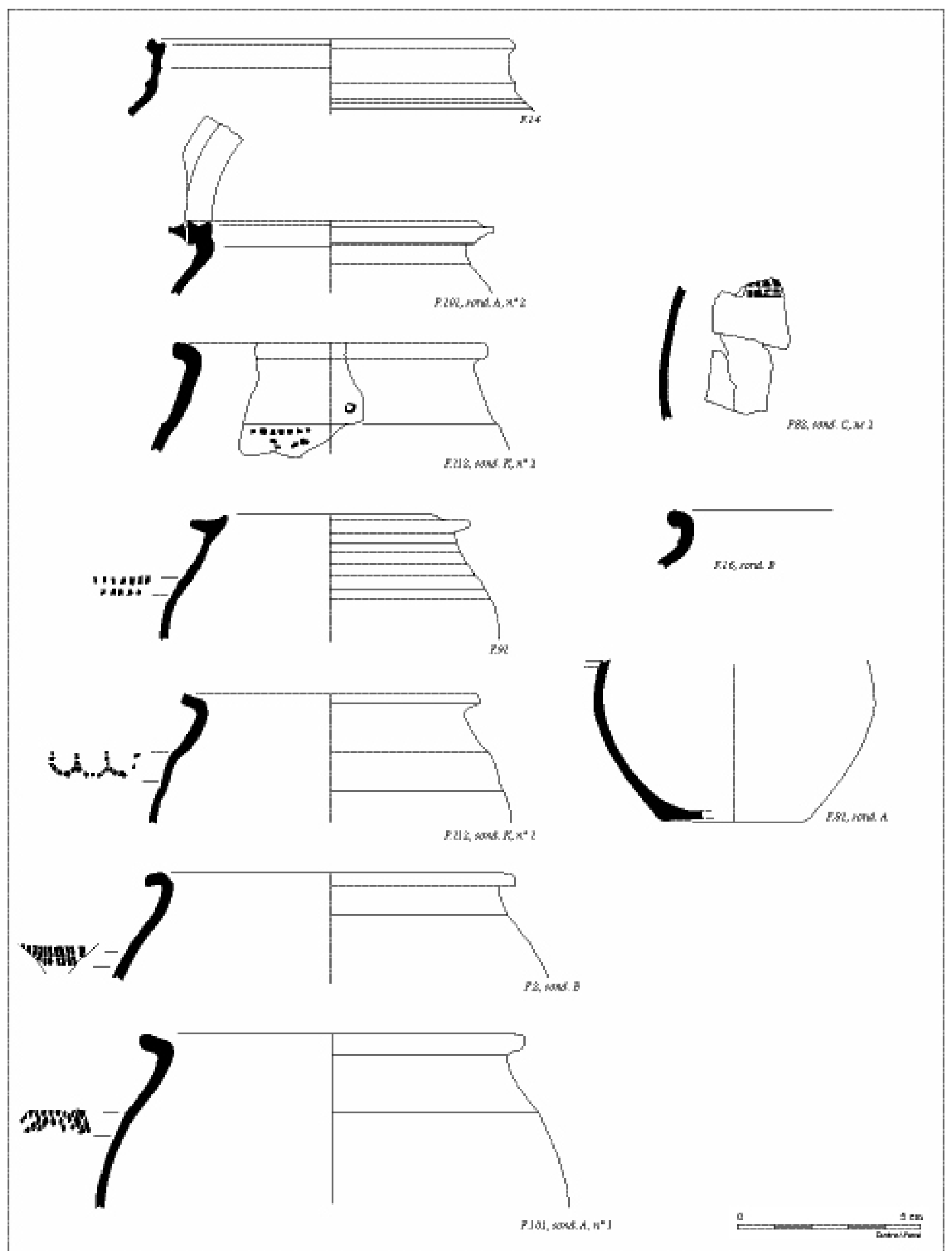

Figure 16 : Céramique du haut Moyen Âge.

Figure 16: Early mediaeval ceramics. 


\section{Divers}

Le mobilier lithique. Parmi les quelques objets retrouvés, un seul a sans doute été utilisé au haut Moyen Âge; il s'agit d'un galet de quartz en demi-sphère qui a pu servir de lissoir (fig. 17). Si tel est bien le cas, ce type d'objet, habituellement en verre, est fréquent sur les habitats du haut Moyen Âge des Pays de la Loire, et plus largement dans toute la moitié nord de la France. Cet outil est généralement associé à l'assouplissement et au repassage des étoffes neuves, en particulier celles fabriquées en lin (Ferdière, 1984, p. 243).

Le reste du mobilier lithique, bien qu'issu de structures du haut Moyen Âge, confirme l'ancienneté de l'occupation néolithique du secteur. Les découvertes se résument à un grattoir sur éclat en silex brun-orangé à fossiles (tertiaire?) issu du fossé $\mathrm{F} 49$ et à un fragment de nucléus à éclats (F 134C) en silex gris (silex de Vion? Identification P. Foré).

Tout aussi exogènes que le silex, trois fragments de calcaire coquillier ont été découverts dans le trou de poteau F 51 situé à proximité de l'entrée principale de l'enclos. La provenance de ces éléments calcaires est difficile à identifier ; On sait que cette roche sert à la fabrication des sarcophages durant la période mérovingienne et que plus tard, aux $\mathrm{XI}^{\mathrm{e}}$ et $\mathrm{XII}^{\mathrm{e}}$ siècles, il est utilisé comme matériau de construction réservé aux éléments de chaînage et aux ouvertures. Il existe par exemple un linteau en calcaire coquillier dans la fenêtre des débuts de l'époque romane qui subsiste au mur sud de l'église d'Azé ou aux ouvertures de la chapelle du Géneteil à Château-Gontier pour le premier quart du XII ${ }^{\mathrm{e}}$ siècle (Valais, 1993, p. 89-115).

Le métal. Quelques fragments ont été recueillis. Ils proviennent principalement de clous en fer.

La faune. (identifications J.-H. Yvinec). Malgré la très forte acidité des sols, quelques éléments de faune ont été recueillis dans les structures du haut Moyen Âge. Bien que rares, ces découvertes permettent néanmoins d'affirmer que nous nous situons bien dans l'environnement immédiat d'un habitat. Elles se résument à quatre dents et fragments
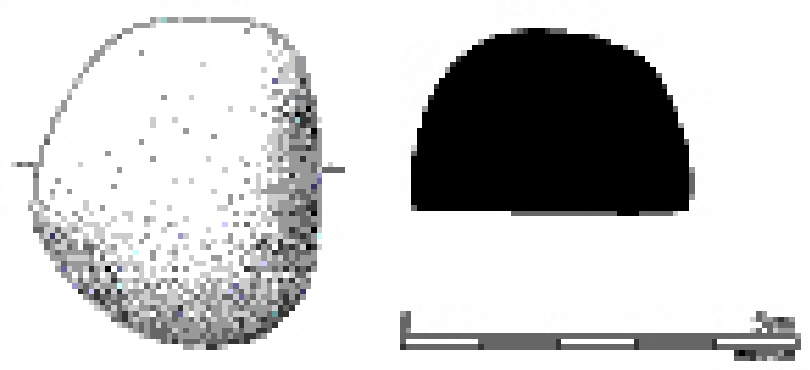

Figure 17 : Un galet de quartz en forme de lissoir en verre. Figure 17: Quartz pebble in shape of a glass smoother. de dents issus des fossés $F 1,51$ et 135 ainsi qu'à un unique fragment d'os long issu du fossé F 15. Il s'agit uniquement de fragments d'os de bovins et parmi les plus résistants qui soient de leur squelette.

\section{SYNTHÈSE ET CONCLUSION}

\section{Vauvert dans le paysage haut médiéval de l'Ouest de la France}

En l'état actuel des connaissances, les sites de comparaison en Pays de la Loire font défaut, les habitats enclos de cette période étant encore rares. On en connaît un exemplaire sur le site des "Vieux Moulins" à Marcé (Viau, 1998) et un second à la "Petite-Vernière » à Trémentines (Bonnin, 2001). Pour ces deux exemples mérovingiens, il s'agit d'enceintes au tracé irrégulier. Il n'en existe qu'un seul exemplaire quadrangulaire, à Trémentines « La Gadellerie " (ibid., p. 66). Partiellement fouillé, il est implanté sur un sommet de versant; il présente également un four et quelques trous de poteaux de la fin du haut du Moyen Âge. Toutefois, la multiplication des enclos à Vauvert évoque peut-être d'avantage le site des Grandes Valinières à SaintSylvain-d'Anjou (Brodeur et Thooris, 1995, p. 167). Avec un phasage général qui reste à établir, ce site du Maine-etLoire montre néanmoins un réseau de fossés orthogonaux dès le haut Moyen Âge.

Il faut aller en Bretagne ou dans le Cotentin pour trouver des sites à enclos multiples qui pourraient présenter le même type de développement que Vauvert. Sur le premier, La Cocherai à Tinténiac (Ille-et-Vilaine), quatre parcelles ont été relevées (Le Boulanger et al., 1992, p. 87-118). Ces enclos, dont trois enserrent des structures d'habitat, occupent de 800 à $2400 \mathrm{~m}^{2}$; faute de phasage précis, le développement du site reste difficile à appréhender. Nous pourrions également ajouter le site mérovingien bas-normand des Agneaux à Bellevue (Manche) qui semble présenter un mode de développement voisin (Carpentier et al., 2003, p. 171189); une des parcelles relevées occupe $1200 \mathrm{~m}^{2}$. À Janzé (Ille-et-Vilaine), les parcelles occupent $1100 \mathrm{~m}^{2}$ en moyenne (Le Boulanger et Leroux, 1999, p. 8).

Le site du Teilleul à Montours (Ille-et-Vilaine) montre également une division géométrique de l'espace en enclos carrés ou rectangulaires. Autour de la parcelle 1 et de son bâtiment se greffent progressivement d'autres enclos (Catteddu [dir.], 2001, p. 97). De plus, la plupart des parcelles, dont certaines sont également pauvres en structures, occupent des surfaces voisines de celles de Vauvert. En dehors de deux enclos qui couvrent $1700 \mathrm{~m}^{2}$ (parcelles 6 et 7), tous les espaces du Teilleul ont des surfaces comprises entre 1000 
et $1500 \mathrm{~m}^{2}$ (ibid., p. 80-84). À Vauvert, ils occupent entre 850 et $1500 \mathrm{~m}^{2}$.

Toutes ces parcelles sont donc proches de l'arpent, qui peut varier à la période carolingienne d'une région à l'autre entre 900 et $1400 \mathrm{~m}^{2}$ (Toati, 1995, p. 26).

\section{Essai d'interprétation}

L'interprétation du site de Vauvert n'est pas sans poser quelques problèmes, le noyau de l'occupation manquant en grande partie.

Les rares structures, le taux de fragmentation de la céramique et les quelques os recueillis évoquent la proximité $\mathrm{du}$ cœur de l'habitat. Cependant, la rareté des trous de poteaux, dont l'attribution chronologique n'est en outre pas assurée, sur un site où les fossés sont pourtant très profonds, milite plutôt pour des espaces enclos réservés aux animaux, à des zones de cultures privilégiées (jardins) ou encore à des activités artisanales (il y a le four F 9 et la fosse complexe F 3).

D'après la dynamique de développement centrifuge des parcelles que nous aurons pu observer (fig. 7), la zone d'habitat se situe très probablement dans l'enclos $\mathrm{A}$ dont les fossés ont livré un mobilier relativement plus abondant qu'ailleurs et surtout les éléments les plus anciens. On peut dès lors expliquer l'implantation du site. Le cour de cet habitat ne se trouvait pas sur le sommet de la colline mais en retrait, sur le rebord occidental d'un plateau, cette implantation constituant, pour la région au moins, une situation beaucoup plus habituelle. L'extension du site aurait progressivement gagné le sommet topographique.

Ces enclos du haut Moyen Âge qui ont alors profondément marqué le paysage local, ne semblent en rien avoir influencé la mise en place d'un nouveau réseau parcellaire auquel sont associés deux chemins et plusieurs fossés. Si nous ne possédons aucun élément de datation sur leur mise en place, une des deux voies était encore utilisée en 1992 (fig. 3).

Il faudra malheureusement attendre qu'un autre habitat mérovingien soit découvert et fouillé, dans son intégralité cette fois, pour que nous puissions valider ou nuancer les hypothèses émises à Vauvert.

Faute de textes suffisamment précis, il est difficile de rattacher de manière certaine Vauvert à la cour de Bazouges, même si la proximité de son église dédiée à Saint Martin $(1,5 \mathrm{~km})$ est à souligner.

D’autre part et plus généralement, le site de Vauvert illustre avec évidence les limites des données offertes par la photographie aérienne et la nécessité de fouiller pour dater avec certitude car, vu d'avion le site, passait pour un enclos de La Tène. En effet, seules les structures situées sur le sommet d'une légère colline et surtout profondément creusées dans le substrat rocheux étaient visibles sur le cliché de 1992. À l'écart des zones où les schistes affleurent, les fossés creusés dans les limons n'apparaissaient plus. La confrontation de la photographie aérienne redressée et du plan de fouille n'apporte pas de données nouvelles hormis que cette superposition montre avec évidence que la fouille s'est finalement limitée à ce qui avait été vu du ciel.

\section{Bibliographie}

ANGot, A., 1900-1910 - Dictionnaire historique, topographique de la Mayenne, Laval, Goupil (4 tomes).

Bocquet, A., Colleter, R. et Valais, A., 2008 - Le cimetière du prieuré de Ménil : étude archéologique, La Mayenne Archéologie Histoire, $\mathrm{n}^{\circ} 30$, p. 78-83.

Bonnin, N. 2001 - Trémentines "La Petite Vernière ", Bilan scientifique régional 1999, Nantes, DRAC des Pays de la Loire, p. 65.

Brodeur, J. et Thooris, C., 1995 - Les Grandes Valinières à Saint-Sylvain-d'Anjou. L'habitat rural du haut Moyen Âge (Actes des XIV ${ }^{\mathrm{e}}$ journées internationales d'Archéologie mérovingienne, Guiry-en-Vexin), Rouen, AFAM, p. 167-174.

Carpentier, V., Ghesquiere, E. et Marcigny, C. (dir.), 2003 Agneaux "Bellevue" (Manche), un établissement rural basnormand du viI ${ }^{\mathrm{e}}$ siècle, Revue archéologique de l'Ouest, 20, p. 171-189.

Cattedu, I. (dir.), 2001 - Les habitats carolingiens de Montours et La Chapelle-Saint-Aubert (Ille-et-Vilaine), Paris, éditions de la Maison des Sciences de l'Homme (coll. « Documents d'Archéologie française ", 89).

Chapelot, J., 1993 - L'Habitat rural, organisation et nature. L'̂llede-France de Clovis à Hugues Capet (Catalogue d'exposition, Musée archéologique du Val d'Oise), éditions du Valhermeil, p. 178-199.

Colleter, R., 2003 - Les cimetières mérovingiens en Mayenne, Société d'Archéologie et d'histoire de la Mayenne, suppl. N ${ }^{\circ} 11$, $208 \mathrm{p}$.

David, F. et Valais, A., 2003 - Un habitat occupé du VII ${ }^{\mathrm{e}}$ au $\mathrm{XII}^{\mathrm{e}}$ siècle : Les Cinq Chemins à Bauné (Maine-et-Loire). Archéologie Médiévale, 33, p. 63-90.

De Broussillon, B., 1903 - Cartulaire de Saint Aubin d'Angers, Paris, Picard, 3 vol.

Demians D’Archimbaud, G., 1981 - Les fouilles de Rougiers, Paris, CNRS, $724 \mathrm{p}$.

Dubillot, X. et Valais, A., 2006 - Les ateliers de potiers du haut Moyen Âge de la Frétellière à Trémentines (Maine-et-Loire). La céramique du haut Moyen Âge dans le nord-ouest de l'Europe ( $V^{e}-X^{e}$ siècle) (Actes du colloque de Caen, 2004), Condé-surNoireau, NEA Du Levant au Ponant, p. 43-64. 
Ferdiere, A., 1984 - Le travail textile en région Centre de l'Âge du Fer au haut Moyen Âge, Revue archéologique du Centre de la France, 23-2, p. 209-265.

Garnotel, A. et Raynaud, Cl., 1996 - Groupés ou dispersés? Les morts de la société rurale en Languedoc oriental (IV $-\mathrm{XII}^{\mathrm{e}}$ siècles), in Galinié, H. et Zadora-Rio, E. (dir.), Archéologie du cimetière chrétien (Actes du $2^{\mathrm{e}}$ colloque ACHEA, Orléans 1994), Revue archéologique du Centre de la France, $11^{\mathrm{e}}$ suppl., p. 139-152.

Guadagnin, R., 1988 - Un Village au temps de Charlemagne. (Catalogue de l'exposition du musée national des ATP), Paris, éditions des Musées nationaux, $357 \mathrm{p}$.

LAmbert, C. et Rioufreyt, J., 1976 - La triple enceinte quadrangulaire de La Tène finale d'Azé près de Château-Gontier, Bulletin de la Commission historique et archéologique de la Mayenne, 41-43, p. 3-32.

LANGLOIS, J.-Y., 2001 - Château-Gontier "Rocade nord ". (Rapport de diagnostic archéologique), Nantes, AFAN et Service régional de l'Archéologie des Pays de la Loire, 27 p.

Le Boulanger, F., Provost, A. et Leroux, G., 1992 - Un village carolingien sur la déviation de la RN 137 à la Cocherais en Tinténiac (Ille-et-Vilaine), Les dossiers du Centre régional d'Archéologie d'Alet, n 20, p. 87-118.

Le Boulanger, F. et Leroux, G., 1999 - Le site de la Tullaye à Janzé (35). Habitat rural en Pays de La Loire et dans les DeuxSèvres (rapport $\mathrm{n}^{\circ} 2 \mathrm{du}$ PCR), Nantes, Service régional de l'Archéologie des Pays de la Loire, p. 8-10.

Legros, S., 2006 - Les prieurés de Château-Gontier et l'établissement d'une seigneurie châtelaine dans le Comté d'Anjou (fin $\mathrm{du} \mathrm{X}^{\mathrm{e}}$ siècle-fin du XI ${ }^{\mathrm{e}}$ siècle), Annales de Bretagne et des Pays de l'Ouest, 113-3, p. 33-59.

MArE, E., 1989 - Église de Bazouges. (Rapport de fouille), Nantes, Service régional de l'Archéologie, 15 p., 50 pl.

Meuret, J.-C., 1993 - Peuplement, pouvoir et paysage sur la marche Anjou-Bretagne (des origines au Moyen Âge), Société d'Archéologie et d'Histoire de la Mayenne, Suppl. N 4, 656 p.

-, 1999 - Site de Vauvert à Château-Gontier (Mayenne). Sondages et évaluation préventifs. Nantes, Sercice régional de l'Archéologie des Pays de la Loire, $45 \mathrm{p}$.

—, 2003 - De l'avion au terrain : données et problèmes de le recherche sur les enclos aux marges de la Bretagne, in MANDY,
B. et de Saulce, A. (dir.), Les Marges de l'Armorique à l'Âge du Fer. (Actes du XXIII colloque de l'AFEAF), Revue archéologique de l'Ouest, Suppl. n 10, p. 23-36.

MeYER, O., 1993 - Un coffre carolingien, L'Tle de France de Clovis à Hugues Capet du ve au X siècle (Catalogue d'exposition, Musée archéologique du Val d'Oise), édit. du Valhermeil, p. 245248.

Morera-Vincotte, I., 2000 - Première synthèse sur les productions céramiques de sites de consommation en milieu rural en Pays de la Loire du $\mathrm{vi}^{\mathrm{e}}$ au $\mathrm{Xv}^{\mathrm{e}}$ siècle, L'Habitat rural au Moyen Âge dans les Pays de la Loire et dans les Deux-Sèvres, (Rapport $\mathrm{n}^{\circ} 3 \mathrm{du}$ PCR), Nantes, Service régional de l'Archéologie des Pays de la Loire, p. 54-76.

Naveau, J., 1992 - Carte Archéologique de la Gaule - La Mayenne 53, Paris, Académie des Inscriptions et Belles-lettres, 176 p.

NAVEAu, J., 1999 - La Mayenne au fil du temps. L'archéologue et le photographe. Laval, Siloé, 120 p.

Peytremann, E., 2003 - Archéologie de l'habitat rural dans le nord de la France du IV au XII siècle, Saint-Germain-en-Laye, Association française d'Archéologie mérovingienne, (Mémoires, XIII), 2 vol., 453 et 442 p.

Petorin, N., Couvin, F. et Morera, I., 1995 - Saint-Georgesdu-Bois (49) "La Sicardière ", (49 280009 AH) (DFS de sauvetage urgent), Nantes, Service régional de l'Archéologie des Pays de la Loire, 96 p.

ToAtı, F.-O. (dir.), 1995 - Vocabulaire historique du Moyen Âge, Paris, La Boutique d'Histoire, 233 p.

Valais, A., 1993 - Premiers édifices romans du Bassin de la Mayenne : éléments de datation, La Mayenne, Archéologie, Histoire, $\mathrm{n}^{\circ} 16$, p. 89-115.

Valais, A. et Dumas, M., 2006 - Courgains "La Colinère ", Diagnostic archéologique. (Rapport final d'opération), Nantes, Service régional de l'Archéologie des Pays de la Loire, 27 p.

Valais, A., Meuret, J.-C. et Nauleau, J.-F. (avec la coll. de Edin, F., Hamon, G. et Mutarelli, V.), 2008 - Le Clos-Henry : une ferme de La Tène finale à Château-Gontier, Revue archéologique de l'Ouest, 25, p. 139-161.

VıAU, Y., 1998 - Les Vieux Moulins à Marcé (Maine-et-Loire). l'Habitat rural au Moyen Âge dans les Pays de la Loire et dans les Deux-Sèvres (Rapport $n^{\circ} 1$ du PCR), Nantes, Service régional de l'Archéologie des Pays de la Loire, p. 54-59. 
